\title{
Optimizing a Pediatric DKA Protocol in a Tertiary Care Hospital System in Northern West Virginia
}

Jennifer L. Burky

jburky@mix.wvu.edu

Follow this and additional works at: https://researchrepository.wvu.edu/etd

Part of the Critical Care Commons, Critical Care Nursing Commons, Endocrinology, Diabetes, and Metabolism Commons, Pediatric Nursing Commons, and the Pediatrics Commons

\section{Recommended Citation}

Burky, Jennifer L., "Optimizing a Pediatric DKA Protocol in a Tertiary Care Hospital System in Northern West Virginia" (2021). Graduate Theses, Dissertations, and Problem Reports. 8256.

https://researchrepository.wvu.edu/etd/8256

This Dissertation is protected by copyright and/or related rights. It has been brought to you by the The Research Repository @ WVU with permission from the rights-holder(s). You are free to use this Dissertation in any way that is permitted by the copyright and related rights legislation that applies to your use. For other uses you must obtain permission from the rights-holder(s) directly, unless additional rights are indicated by a Creative Commons license in the record and/ or on the work itself. This Dissertation has been accepted for inclusion in WVU Graduate Theses, Dissertations, and Problem Reports collection by an authorized administrator of The Research Repository @ WVU.

For more information, please contact researchrepository@mail.wvu.edu. 
Graduate Theses, Dissertations, and Problem Reports

2021

Optimizing a Pediatric DKA Protocol in a Tertiary Care Hospital System in Northern West Virginia

Jennifer L. Burky 
Optimizing a Pediatric DKA Protocol in a Tertiary Care Hospital System in Northern West

\author{
Virginia \\ Jennifer L. Burky \\ Project Report submitted \\ to the School of Nursing \\ at West Virginia University
}

In partial fulfillment of the requirements for the degree of
Doctor of Nursing Practice

Kendra Barker, DNP, Chair

Charles Mullett, MD, Ph.D.

Lya Cartwright-Stroupe, DNP

Department of Nursing

Morgantown, WV 2021

Keywords: diabetic ketoacidosis, pediatric, diabetes, protocol, algorithm, nursing

Copyright 2021 Jennifer Burky 


\section{Abstract \\ Optimizing a Pediatric DKA Protocol in a Tertiary Care Hospital System in Northern West Virginia}

\section{Jennifer Burky, MSN, APRN-CNP, FNP-BC}

Background: Diabetic ketoacidosis (DKA) is a serious complication of diabetes that occurs when the body compensates for insufficient insulin, producing dangerously high levels of ketones. Without proper treatment, DKA can be fatal. For that reason, staff in the pediatric intensive care unit (PICU) at a tertiary care hospital in northern West Virginia expressed concerns about the complexity of and lack of adherence to a poorly referenced and dated DKA treatment protocol.

Objective: The purpose of this project was to assess the feasibility and sustainability of optimizing the DKA protocol in the PICU. The primary objective was to implement an updated, simplified, evidence-based DKA protocol in the PICU to promote adherence by staff members.

Methods: A literature search was performed based on the PICOT question "In pediatric patients, how does using a protocol to manage DKA compared to not using a protocol affect patient outcomes throughout hospital admission?" A total of 26 articles were used: 6 that directly supported implementation of a DKA protocol, and 20 that supported various aspects of DKA management that were essential for an updated, evidence-based protocol. Based on these, updates were made to the existing protocol to make it easier to use but still conform to clinical guidelines set forth by the International Society for Pediatric and Adolescent Diabetes (ISPAD). The Duffy Quality-Care Model and the Star Model of Knowledge Transformation were the theoretical frameworks for this project. Focus groups were used to assess demand and practicality for the protocol changes and assimilate the evidence-based guidelines into the revised protocol. Staff was educated to the changes, and the revised protocol was implemented for a 90-day trial period. Staff satisfaction and protocol usage evaluation were evaluated through staff surveys with both quantitative Likert scale data and qualitative open-ended question data. Rapid cycle quality improvement strategies of staff interviewing and survey data analysis were used to troubleshoot protocol issues during the trial period.

Results: Using evidence-based literature, a fully optimized DKA protocol was successfully developed and implemented in the PICU. Feasibility of long-term sustainability was confirmed by staff surveys with $78.9 \%$ reporting they were likely to use the new protocol. There was report through the interview and open-ended question answers that patient glucose levels remained high longer with the new protocol, though the acidosis still corrected without issue, leading to the identification of a missed logic step in the protocol algorithm for rebound hyperglycemia during the dextrose infusion. There were no medical complications related to this missed step, but it was identified as an area of the protocol needing further development.

Discussion: Though there was a high percentage of staff members reporting they were likely to use the new protocol, there were mixed results for whether the old or new protocol was preferred. The missed step in the algorithm for rebound hyperglycemia with the dextrose 
infusion is under review by the PICU medical staff, but recommendations have not yet been determined for appropriate correction as this rebound hyperglycemia also has some protective effects against hypoglycemia during acidosis correction. Attention to this issue with the algorithm will likely improve staff satisfaction with the protocol.

Conclusion: At present, the PICU staff plan a sustained adoption of the revised DKA protocol. Using an algorithmic, evidence-based DKA protocol standardizes care provided to patients, reducing morbidity and mortality and promoting patient safety and improved patient outcomes. 


\section{Acknowledgements}

First and foremost, I want to thank GOD, without whom none of this would be possible. Second, I want to thank my family and friends for their support and understanding when I had to miss events, or I was distracted because of my project.

Next, I want to thank my committee. Dr. Kendra Barker, my Faculty of Record, without you there is absolutely no way this project would have been completed. You encouraged me when I did not want to keep going. You helped me find words when I was at a loss. You helped me see solutions to project problems when I was too immersed in them to see the big picture. Dr. Charles Mullett, thank you for your wisdom and expertise in navigating the infrastructure of the PICU. I do not think the protocol would have been as well-received if not for you. Dr. Lya Stroupe, thank you for helping me navigate the infrastructure of the Research Committee and showing me where to find answers when I thought I had exhausted my resources.

In addition, I would like to thank Kevin Steurer, the PICU Nursing Champion, who willingly acted as my eyes and ears of the nursing staff since I could not be there due to pandemic restrictions in place. He made sure that the nurses received my emails and surveys and helped to coordinate the education sessions through WebEx. I would also like to thank Tiffany Schwalm, the nurse manager of the PICU, for hanging educational posters about the protocol around the unit and for allowing me the opportunity to implement this quality improvement change in the PICU.

Finally, I wish to thank the PICU staff for allowing me to make changes to their busy routines. I appreciate your patience and your willingness to try something new. 
Table of Contents $\quad$ V

Acknowledgements $\quad$ iv

$\begin{array}{ll}\text { Introduction } & 1\end{array}$

$\begin{array}{ll}\text { Background } & 1\end{array}$

$\begin{array}{ll}\text { Problem Statement } & 4\end{array}$

$\begin{array}{ll}\text { Purpose of Project } & 4\end{array}$

$\begin{array}{ll}\text { Significance of Project } & 4\end{array}$

$\begin{array}{ll}\text { Literature Search } & 5\end{array}$

$\begin{array}{ll}\text { Literature Review } & 7\end{array}$

$\begin{array}{ll}\text { Literature Synthesis } & 14\end{array}$

$\begin{array}{ll}\text { Theoretical Framework } & 19\end{array}$

$\begin{array}{ll}\text { Project } & 21\end{array}$

Congruence to Organization's Strategic Plan 21

Evidence of Key Site Support $\quad 22$

Organizational and Project Needs Assessment 22

Identification of Project Resources 23

$\begin{array}{ll}\text { Personnel } & 23\end{array}$

$\begin{array}{ll}\text { Materials } & 23\end{array}$

$\begin{array}{ll}\text { Technology } & 24\end{array}$

$\begin{array}{ll}\text { Strategic Analysis } & 24\end{array}$

$\begin{array}{ll}\text { Feasibility Analysis } & 25\end{array}$

$\begin{array}{lr}\text { Market Analysis } & 26\end{array}$

$\begin{array}{ll}\text { Sales and Marketing } & 27\end{array}$ 
Operations and Financial Plan

$\begin{array}{ll}\text { Description and Design } & 28\end{array}$

$\begin{array}{ll}\text { Measurable Project Objectives } & 30\end{array}$

Timeline of Project 31

$\begin{array}{ll}\text { Results } & 32\end{array}$

$\begin{array}{ll}\text { Discussion } & 38\end{array}$

$\begin{array}{ll}\text { Recommendations } & 41\end{array}$

$\begin{array}{ll}\text { Attainment of DNP Essentials } & 42\end{array}$

$\begin{array}{ll}\text { Summary } & 46\end{array}$

$\begin{array}{ll}\text { Appendices } & 47\end{array}$

A - Star Model of Knowledge Transformation 47

B- Organization Letter of Support $\quad 48$

$\begin{array}{ll}\text { C - Budget Plan } & 49\end{array}$

D1 - Clinical Practice Guidelines 2018 Algorithm found in Wolfsdorf et al., 201851

D2 - Previous PICU DKA Protocol 52

D3 - Calculations for Fluid Maintenance and Replacement Values Chart 56

D4 - Revised PICU DKA Protocol 57

E - Staff Survey $\quad 62$

F1 - Project Timeline $\quad 65$

F2 - Project Timeline Table $\quad 66$

G - Survey Results Graphs

$\mathrm{H}$ - Themes of Open-Ended Questions of Surveys 71

$\begin{array}{ll}\text { References } & 75\end{array}$ 


\section{Introduction}

This project was designed to implement an evidence-based practice change for diabetic ketoacidosis (DKA) management of pediatric patients at a Magnet-designated tertiary academic medical center in northern West Virginia (WV). Though this hospital had a basic DKA protocol in use that had been in effect for over a decade, the protocol had not been updated in several years. The protocol was poorly referenced and undated, so it was difficult to determine what the evidence base was for the protocol when it was written. In addition, the 4-page, heavily detailed paper protocol in use was described as "too long" and "difficult to follow" by staff. To provide the highest quality of care to patients, it was important to revise the protocol to be compliant to the most current guidelines and simple to use and follow. The frameworks for this practice change were the Star Model of Knowledge Transformation (Stevens, 2012) and the Duffy Quality-Caring Model (Duffy \& Hoskins, 2003). The medical changes for the protocol were based on the algorithm for the management of diabetic ketoacidosis identified in the International Society for Pediatric and Adolescent Diabetes (ISPAD) Clinical Practice Consensus Guidelines (Wolfsdorf et al., 2018). This practice change was designed for healthcare providers in West Virginia who treat pediatric patients with DKA, primarily those who work in a Pediatric Intensive Care Unit in a tertiary care hospital in northern WV.

\section{Background}

Diabetic ketoacidosis (DKA) is defined as a serious complication of diabetes that occurs when the body begins to produce high levels of blood acids called ketones in response to insufficient insulin that facilitates the transfer of glucose into cells. Without insulin, the body begins to break down fat as fuel, creating ketones. These ketone levels rise in the blood, which increases blood acidity and creates ketoacidosis, requiring intervention. DKA is identified as a 
"life-threatening but preventable complication of diabetes" (Benoit, Zhang, Geiss, Gregg, \& Albright, 2018). It is generally characterized by hyperglycemia (blood glucose greater than or equal to $200 \mathrm{mg} / \mathrm{dL}$ ), a venous $\mathrm{pH}$ less than 7.3 or serum bicarbonate less than $15 \mathrm{mmol} / \mathrm{L}$, and ketonemia (blood beta-hydroxybutyrate of $3 \mathrm{mmol} / \mathrm{L}$ or greater) or moderate to large ketonuria (Wolfsdorf et al., 2018). Due to the mechanisms in which the body attempts to compensate for lack of insulin, hyperglycemia, and the resulting chemical imbalances, the patient may display any combination of the clinical signs of dehydration, tachycardia, tachypnea and deep sighing breaths (Kussmaul breathing), breath smelling of acetone, nausea and/or vomiting, abdominal pain, blurry vision, confusion, drowsiness, and progressive decrease in mental status that, left untreated, can deteriorate to coma and death (Wolfsdorf et al., 2018).

Significant morbidity and mortality associated with DKA in pediatric patients is usually associated with cerebral edema, although this occurs in less than $1 \%$ of cases of DKA (Patel et al., 2015). There are recognized risk factors that contribute to the development of cerebral edema, although the etiology is poorly understood. Wolfsdorf et al. (2018) lists the risk factors for cerebral edema as elevated serum urea nitrogen concentration, severe acidosis, and hypocapnia. Skitch and Valani (2015) state that risks are two-fold: patient-related variables such as younger age, new diagnosis of diabetes, and greater illness severity as evidenced by lower baseline bicarbonate, higher serum urea, and lower partial pressure of arterial carbon dioxide; and treatment-related variables such as administration of bicarbonate, administration of large volumes of fluids within the first four hours of treatment, and administration of insulin within the first hour of treatment. Vavilala et al. (2010) investigated blood brain barrier permeability during DKA in children and found an increase in blood brain barrier permeability that they postulated could be attributed to hyperglycemia, matrix metalloproteinase activity, insulin administration, 
and inflammation as had been demonstrated in earlier animal studies. Vavilala et al (2010) suggested that vasogenic cerebral edema could be caused by increased cerebral blood flow associated with early DKA treatment (fluid resuscitation after dehydration), increased blood brain barrier permeability, or both. Ma et al. (2014) found that pediatric patients with DKA showed signs of impaired cerebral autoregulation compared to other children with type I diabetes mellitus who were not experiencing DKA, though it was uncertain if the impaired autoregulation caused cerebral edema or cerebral edema caused the impairment.

Regardless of the underlying pathophysiological cause of cerebral edema in pediatric patients, it appears that the arbitrary administration of fluids affects brain swelling. A study completed by Bakes et al. (2016), showed that a higher volume of fluids decreased time of correction of acidosis in DKA but noted that the majority of patients who experienced cerebral edema had received more isotonic fluids in the first 8 hours of treatment compared to those who did not. Fluid resuscitation, therefore, is an important component of management for DKA, as well as an essential updated evidence factor to implement into a modernized, evidence-based DKA protocol. Both prevention and early recognition of cerebral edema as a complication of DKA are important measures for better patient outcomes and safety and are necessary components of an optimized DKA protocol.

Though DKA can occur in either type 1 diabetes mellitus (T1DM) or type 2 diabetes mellitus (T2DM), it is commonly associated with T1DM. DKA is the presenting manifestation of a T1DM diagnosis in 25-30\% of patients with T1DM (Desai, Mehta, Mathias, Menon, \& Schubart, 2018). DKA can occur at any age, but pediatric patients tend to be more susceptible to it than adults, and younger children are more susceptible to complications associated with DKA. In fact, Wolfsdorf et al. (2018) lists younger age as a risk factor for developing DKA. Between 
the years of 2002 and 2012, approximately 246,925 patients under the age of 18 were hospitalized for DKA in the United States (Patel et al., 2015). At the site of this project, the local context of the problem was investigated. The pediatric intensive care unit (PICU) in a tertiary care hospital in northern WV provided care for 187 pediatric patients with a diagnosis of diabetic ketoacidosis between January 1, 2017 and January 1, 2019 according to an electronic audit of diagnosis codes in the electronic medical record (EMR) used (Epic).

\section{Problem Statement}

Staff in the PICU at a tertiary care hospital in northern WV expressed concerns about the complexity of and lack of adherence to a poorly referenced and dated DKA treatment protocol being used within the PICU to treat approximately 100 cases of DKA per year.

\section{Purpose of the Project}

The purpose of this project was to revise, update, and implement the protocol used in the PICU for treating patients with DKA.

\section{Significance of the Project}

Diabetic ketoacidosis (DKA) is a serious and expensive complication of uncontrolled diabetes, with impact on morbidity and mortality as well as healthcare costs. The number of individuals diagnosed with diabetes and DKA continues to rise annually. According to MayerDavis et al. (2017), 11,245 youths aged 0-19 years in the United States were identified to have type 1 diabetes between 2002 and 2012. During that 10-year period the incidence of type 1 diabetes diagnosis in the United States increased from 19.5 cases per 100,000 youths per year during 2002-2003 to 21.7 cases per 100,000 youths per year during 2011-2012 (Mayer-Davis et al., 2017).Benoit et al. (2018) found that the rate of hospitalizations for DKA increased by 54.9\% between 2009 and 2014, and the highest rates were among people less than 45 years. According 
to Desai et al. (2018), 20,705 children under the age of 17 years in the United States were diagnosed with DKA in 2014.

Some complications related to DKA are electrolyte abnormalities like hyponatremia, hyperchloremia and hypokalemia; shock; acute kidney injury; heart arrhythmias related to the electrolyte imbalances and dehydration; and cerebral edema (Abbas, Arbab, Anwar ul Haque, \& Humayan, 2018). Though cerebral edema is a rare complication, an article by Patel et al. (2016) cited the mortality rate of children with cerebral edema was $40 \%$. Additionally, time spent hospitalized for DKA impacted morbidity through time lost at school for youths with this condition and work for their parents.

According to Desai et al. (2018), one admission for DKA costed approximately $\$ 26,566$ in 2014, and the total aggregate cost for DKA hospitalizations for the US in 2014 was $\$ 5.1$ billion. Therefore, in 2 years, pediatric DKA patients treated in the PICU cost approximately $\$ 4,967,842$ in healthcare costs. As such, it is important to minimize these expenses and decrease complications by using a protocol that follows clinical guidelines and is simple to understand and follow as early in the patient treatment plan as possible.

\section{Literature Search}

Multiple searches were conducted through EbscoHost that included the databases Academic Search Complete, Alt HealthWatch, Commonwealth Agricultural Bureaux (CAB) Abstracts 1990-Present, Cumulative Index of Nursing and Allied Health Literature (CINAHL) with Full Text, Education Resources Information Center (ERIC), Health Source-Consumer Edition, HealthSource: Nursing/Academic Edition, International Pharmaceutical Abstracts, Medical Literature Analysis and Retrieval System Online (MEDLINE or MEDLARS online), and TOPICsearch. Restrictions placed were English language, Peer Reviewed, Human subjects, 
Child (0-18 years), and Published 2015-2019. Keywords searched were diabetic ketoacidosis AND pediatrics OR children ( 40 results, 8 after abstract evaluated), diabetic ketoacidosis AND subcutaneous insulin AND pediatrics (11 results, 3 after abstract evaluated), diabetic ketoacidosis AND balanced electrolyte solution AND pediatrics (0 results), and diabetic ketoacidosis AND balanced electrolyte solution (1 result).

Pubmed was searched using keywords diabetic ketoacidosis AND pediatric, with 729 results. Restrictions of publication date within the last 5 years (for most up-to-date information), human species, English language, and child from birth to 18 years were placed with 159 results, 9 of which were used after abstract evaluation.

Cochrane library was searched with and without Medical Subject Headings (MeSH) terms. Initially, the keywords were pediatric diabetic ketoacidosis treatment, which yielded 49 results. Restriction of publication date between 2015 and 2019 narrowed the results to 39; 3 articles were used after abstract evaluation. Searching by MeSH descriptor diabetic ketoacidosis with qualifier of therapy yielded 12 results. Restriction of publication date between 2015 and 2019 yielded 5 results; 3 were repeated articles and the other 2 were eliminated after abstract review.

Google Scholar was searched with keywords pediatric diabetic ketoacidosis AND balanced electrolyte solution, yielding 1,230 and "pediatric diabetic ketoacidosis" AND balanced electrolyte solution, which yielded 41 results. With restrictions of published after 2015 and deleting articles with adult in them, there were 16 results; 3 of those were repeated articles and the other 13 were not relevant to the topic.

Two articles were found through the snowballing technique. The article by Shankar, Haque, Churchwell, and Russell (2007) was found as a reference for the article by Barski, 
Brandstaetter, Sagy, and Jotkowitz (2018). The article by Koves et al. (2014) was found as a reference within the article by Horvat et al. (2018).

Of 273 articles found after restrictions were placed, evaluation of the abstracts yielded a total of 26 articles used, 6 of which directly supported implementation of a DKA protocol, and the other 20 support various aspects of DKA management that are essential for a modernized evidence-based protocol.

\section{Literature Review}

During the literature search 26 articles were found pertaining to the management of DKA in pediatric patients. Those discussed are the 6 that directly supported the implementation of a DKA protocol (Wolfsdorf et al., 2018), (Koves et al, 2014), (Horvat et al., 2018), (Peeters et al, 2015), (Nemri et al., 2017), and (Flood et al., 2019); then one that compares various protocols for consistencies in treatment (Skitch \&Valvani, 2015), and finally a randomized control trial comparing differing maintenance fluids and volumes (Kupperman et al., 2018). Articles are arranged so those validating the use of a protocol appear first. The articles are then arranged by strength of evidence.

A panel of expert physicians from the International Society for Pediatric and Adolescent Diabetes (ISPAD), Lawson Wilkins Pediatric Endocrine Society (LWPES), and the European Society of Pediatric Endocrinology (ESPE) released a consensus of clinical guidelines in 2018 for the management of DKA (Wolfsdorf et al., 2018). This consensus incorporates recommendations based on results from the Pediatric Emergency Care Applied Research Network (PECARN) fluid study (Kuppermann et al., 2018), which will be discussed later in this section. Included in the article by Wolfsdorf et al. (2018) are the definition and pathophysiology, risk factors, recognition of symptoms, emergency assessment (which alludes to PALS 
recommendations), management of DKA from initial diagnosis through treatment after resolution of acidosis (introduction of oral fluids and subcutaneous insulin), comorbidities and mortality rates, decreasing risks for life-threatening comorbidities like cerebral edema, comparing DKA to hyperglycemic hyperosmolar state and discussing differences in treatment, and steps to prevent future episodes of DKA. Wolfsdorf et al. (2018) provides a treatment algorithm that sets forth the framework for the management of DKA in pediatric patients.

Koves et al. (2014) compiled a systematic review of published literature to implement an evidence-based clinical pathway for the treatment of DKA in pediatric patients. This was executed at Seattle Children's Hospital, a 350-bed children's hospital where approximate 150 patients are hospitalized annually for DKA management over a two-year span (Koves et al., 2014). After implementing the clinical pathway through electronic order sets and paper clinical pathways, Koves et al. (2014) found that though ICU hospitalizations for DKA increased (34\% pre-implementation to $51 \%$ the first year after implementation to $39 \%$ the second year), the length of time spent in the ICU was decreased by 1.06 days in the first year compared to preimplementation and 0.69 days in the second year compared to pre-implementation. Koves et al. (2014) also found the incidence of cerebral edema decreased from $2.9 \%$ pre-implementation to $1.8 \%$ in the first year after implementation and $1.2 \%$ in the second year. The implementation of the clinical pathway and procedures for management also allowed for the identification of an issue with hypokalemia that the multidisciplinary team was able to rectify within the clinical pathway, decreasing the amount of time before patients received potassium replacement salts from a mean time of 90 minutes from beginning of treatment to a mean time of 19 minutes (Koves et al., 2014). Overall, this article demonstrates that implementation of an evidence-based DKA protocol decreases time spent in the hospital, helps identify and address acute treatment 
needs (i.e. hypokalemia) of patients more efficiently, and decreases the incidence of cerebral edema.

Horvat et al. (2018) conducted a retrospective cohort study to identify temporal trends in treatment-related outcomes surrounding the implementation of a standardized management pathway, or protocol. Horvat et al. (2018) found treatment-related outcomes to improve after the implementation of a performance improvement intervention that mandated intensive care unit admission and standardization of a management pathway. This cohort study evaluated 1,225 children with DKA through electronic health record, and outcomes were measured based on incidence of cerebral edema, hypoglycemia, severe hyperchloremia, head computed tomography (CT) utilization, and central venous line placements (Horvat et al., 2018). Though regression analysis showed no change in incidence of cerebral edema or hypoglycemia from before and after implementation of the intervention, there was a decrease in the utilization of head CT and also in the incidence of severe hyperchloremia (Horvat et al., 2018). Horvat et al. (2018) attributed the decline in hyperchloremia to the replacement of potassium chloride with potassium acetate or potassium phosphate as well as some utilization of $0.45 \%$ saline with the addition of dextrose during management. Horvat et al. (2018) noted that there was a decreased length of stay after the implementation of the intervention, possibly due to a decrease in practice variability. Variability was decreased by limiting treatment to the ICU (fewer interventionalists so less variability) and the implementation of a standardized management pathway (Horvat et al., 2018). Horvat et al. (2018) identified associations of presenting $\mathrm{pH}, \mathrm{pCO}_{2}, \mathrm{BUN}$, and systolic blood pressure with cerebral edema, and states these were factors related to cerebral edema identified in other studies. Overall, this study demonstrates that the implementation of a DKA protocol decreases length of hospital stay and reduces variability of treatment. 
Peeters et al. (2015) proposed that pediatric DKA could be managed in a community hospital setting outside of a high dependency unit with a precise DKA management protocol and experienced staff and a consultant pediatrician. Peeters et al. (2015) conducted a descriptive cohort study in a community general hospital using 95 pediatric patients who, between 2001 and 2010, were admitted and treated for severe DKA as defined by initial $\mathrm{pH}$ of less than 7.3 and/or bicarbonate less than $15 \mathrm{mmol} / \mathrm{L}$ with hyperglycemia and dehydration. Children who were transferred to another hospital during treatment or whose initial $\mathrm{pH}$ was greater than 7.3 were excluded from the study (Peeters et al., 2015). There was no incidence of cerebral edema, 6 hypoglycemic events in 3 separate patients, severe hypokalemia of $2.2 \mathrm{mmol} / \mathrm{L}$ in $1 \mathrm{case}, 28$ incidences of potassium levels between 2.6 and $3.5 \mathrm{mmol} / \mathrm{L}$, and a mean time of recovery to a $\mathrm{pH}$ of at least 7.3 that was 665 minutes, or slightly more than 11 hours (Peeters et al., 2015). Peeters et al. (2015) did recommend a lower rate of insulin $(0.05 \mathrm{U} / \mathrm{kg} / \mathrm{hour})$ to avoid hypoglycemic episodes; they stated that there was limited evidence that a higher dose of insulin led to a quicker resolution of lipolysis and ketogenesis. Peeters et al. (2015) listed the small size of their study as a limitation. Overall, this study claims that DKA can be safely treated in a community hospital without tertiary pediatric care available if an evidence-based protocol is in place and the institution has staff experienced in treating pediatric patients experiencing DKA.

Nemri et al. (2017) examined the effect of implementing clinical practice guidelines in treating DKA on length of hospital stay. This case-control retrospective study was conducted at King Khalid University Hospital, King Saud University Medical City, Riyadh, Saudi Arabia from January 2008 through January 2014 (Nemri et al., 2017). It compared 63 episodes of DKA with 41 patients after implementation of clinical guidelines with 40 episodes of DKA in 33 patients treated before the implementation of the guidelines (Nemri et al., 2017). Pre- 
implementation hospital stays were 107.4 +/- 65.6 hours compared to $68.6+/-53.1$ hours after implementation of guidelines (Nemri et al., 2017). The aim was to standardize the management of DKA and decrease variations between physicians (Nemri et al., 2017). Nemri et al. (2017) report the improvement in efficiency of the management of DKA is at least partially due to the implementation of the clinical guidelines but also emphasize the importance of educating the patients and their families as well. Overall, this study shows that the implementation of an evidence-based DKA protocol based on clinical guidelines decreases the length of hospital stays and helps to standardize the care provided to the patients.

Flood et al. (2019) offers a template for implementation of a new DKA protocol, or order set, in a Canadian pediatric tertiary care center. In this quality-improvement case study in a pediatric tertiary care center that sees approximately 30 DKA admissions per year, a collaborative multidisciplinary team from pediatric endocrinology, PICU, pediatric emergency department, general pediatrics, pediatric residents, pediatric nurses and nurse educators, pediatric clinical pharmacy and laboratory medicine formulated an order set based on literature published both nationally and internationally (Flood et al., 2019). The order set was implemented through a plan-do-study-act approach, and it was evaluated by comparing to previous patient records in order to adjust treatment for both end-users' needs and clinical concerns (Flood et al., 2019). After implementation, 30 DKA episodes involving 29 different patients were evaluated for compliance to the order set and showed that the implementation of the order set showed early signs of improvement of DKA management in the pediatric patient population (Flood et al., 2019). Initial IV fluid bolus prior to initiation of insulin were received in $96.7 \%$ of patient as opposed to $92 \%$ before implementation (Flood et al., 2019). Initiation of IV fluids containing 40 $\mathrm{mmol} / \mathrm{L}$ of potassium chloride improved from $40 \%$ to $79.3 \%(\mathrm{p}=0.009)$, though there was no 
difference in incidence of moderate or severe hypokalemia (Flood et al., 2019). Dextrose was started once glucose levels reached $17 \mathrm{mmol} / \mathrm{L}$ (about $300 \mathrm{mg} / \mathrm{dL}$ ) $93.1 \%$ of the time after initiation of the order set, compared to $67.4 \%$ prior to initiation $(\mathrm{p}=0.009)$, though incidence of hypoglycemia showed no statistically significant difference (Flood et al., 2019). Some limitations listed were related to physician buy-in and physician practice change, lack of pointof-care access to the order set, and general awareness of the presence of the order set (Flood et al., 2019). Overall, this study showed that the implementation of an order set improved DKA management in the pediatric patient population, and specifically showed improved adherence for early dextrose infusion.

Skitch and Valani (2015) compared protocols used at 13 different children's hospitals throughout Canada to identify similarities and variances. Consistencies found between protocols included to infuse insulin intravenously continuously at a rate of 0.1 units $/ \mathrm{kg} /$ hour after initial fluid resuscitation; to incorporate $40 \mathrm{mEq} / \mathrm{L}$ of potassium chloride into maintenance fluids after the patient is no longer hyperkalemic (potassium level greater than $5-5.5 \mathrm{mmol} / \mathrm{L}$ ); to frequently assess electrolyte levels; to monitor blood glucose hourly; and to add dextrose to fluids to prevent hypoglycemia (Skitch \& Valani, 2015). Variations in protocols centered around fluid administration, whether it was the amount and rate of the initial bolus or the hourly rate for the maintenance fluid (Skitch \& Valani, 2015). The other variation involved bicarbonate administration; five treatment protocols stated to never give bicarbonate, one protocol did not give recommendations at all regarding bicarbonate, and the remaining protocols recommended bicarbonate administration only in severe acidosis $(\mathrm{pH}<6.9)$ (Skitch \& Valani, 2015). Skitch and Valani (2015) identified as a limitation the fact that they only evaluated protocols and not actual practices within facilities. Another limitation was that they only evaluated protocols used 
within pediatric tertiary care facilities (Skitch \&Valani, 2015). Skitch and Valani (2015) recognized that many pediatric patients are initially treated in non-pediatric emergency departments and were often given double the amount of a resuscitation bolus as was given to patients who were treated in a pediatric emergency department. Despite the excess fluid, Skitch and Valani (2015) mention that there was no higher occurrence of cerebral edema in those patients given the larger boluses than those who were treated according to guidelines and recommend further research of how adherence to protocols affects outcomes of patients. Overall, this study showed that the 13 children's hospitals evaluated had similar protocols for most aspects of treatments except fluid administration and the administration of bicarbonate in emergent situations.

A study by Kuppermann et al. (2018) is worth noting because while it does not address the implementation of a pediatric DKA protocol directly, it was a randomized, controlled trial (RCT) that investigated a common issue not agreed upon by experts: the effects of rate of administration and sodium chloride content of intravenous fluids on the neurological outcomes of pediatric patients treated for DKA. This RCT was conducted throughout 13 emergency departments in the Pediatric Emergency Care Applied Research Network (PECARN) and included 1389 episodes of DKA in 1255 children (Kuppermann et al., 2018). Participants were randomly placed in one of four groups: fast rate of rehydration with $0.45 \%$ sodium chloride, fast rate of rehydration with $0.9 \%$ sodium chloride, slow rate of rehydration with $0.45 \%$ sodium chloride, and slow rate of rehydration with $0.9 \%$ sodium chloride; all other treatment for all four groups was identical (Kuppermann et al., 2018). Patients and their families did not know to which group they belonged, but it was necessary for the care team to know in order to follow the correct protocol (Kuppermann et al., 2018). Neurological status was evaluated using the 
Glasgow Coma Scale (GCS) to judge mental status changes (defined as at least consecutive GCS scores less than 14 during any hour within the first 24 hours of treatment), incidence of clinically-apparent brain injury during treatment, short-term memory assessments during treatment, and memory and IQ 2 and 6 months after treatment (Kuppermann et al., 2018). During treatment in 48 episodes the GCS declined to less than 14; 22 episodes resulted in receiving hyperosmolar therapy for suspected cerebral edema; and 12 episodes of children with clinically apparent brain injury (Kuppermann et al., 2018). There were no statistically significant differences between groups for decline in GCS (Kuppermann et al., 2018). Though there was higher incidence of clinically apparent brain injury in the slow rehydration groups than the fast rehydration groups, the difference was not significant (Kuppermann et al., 2018). Incidence of hypoglycemia and time to resolution was similar in all four groups (Kuppermann et al., 2018).

Kuppermann et al. (2018) reported no significant differences in neurocognitive outcomes after recovery, but there were some non-neurological adverse effects worth noting. Kuppermann et al. (2018) reported more hyperchloremic acidosis in the groups receiving $0.9 \%$ sodium chloride than those receiving $0.45 \%$ and also in groups receiving rapid rehydration more than in groups receiving slow rehydration. Hypocalcemia and hypophosphatemia were more prevalent in groups receiving $0.9 \%$ sodium chloride, and those receiving the rapid rate had higher incidence of hypocalcemia but not hypophosphatemia (Kuppermann et al., 2018). Overall, the study showed no significant difference in neurological changes noted when different concentrations and volumes of sodium chloride were used for treatment, but there were more non-neurological adverse effects when $0.9 \%$ sodium chloride was used compared to $0.45 \%$ sodium chloride and in the rapid rehydration groups versus the slow rehydration groups.

\section{Literature Synthesis}


One may argue that the existence of research in different treatment parameters is an indication for the support of some form of guidelines. Though experts concur that guidelines are needed, there is some agreement and some argument over what is needed. Universally, the research agrees that DKA generally presents with some level of dehydration and acidosis related to ketonemia caused by deficient insulin and that the treatment is IV fluids, insulin, and replacement of depleted electrolytes. However, the methods vary. While the majority of the research advises to treat initially with a bolus of $10-20 \mathrm{ml} / \mathrm{kg}$ of fluid over 1 hour and then continue maintenance fluids to correct dehydration (Bakes et al., 2017), (Kim, 2015), (Kuppermann et al., 2018), and (Wolfsdorf et al., 2018), there is some dispute over which fluids to use and at what rate to infuse them. The clinical guidelines provided by Wolfsdorf et al. (2018) recommend to give the initial bolus of $10 \mathrm{ml} / \mathrm{kg}$ of $0.9 \%$ sodium chloride (i.e. normal saline) over 30 to 60 minutes and repeat as necessary if the patient is not in shock, then follow with $0.45 \%$ sodium chloride solution or a balanced salt solution, which includes Ringer's lactate, Hartman's solution, or Plasmalyte in order to decrease the incidence of hyperchloremic metabolic acidosis (Wolfsdorf et al., 2018). The Annals of Pediatric Endocrine and Metabolism (APEM) correlate with these suggestions (Kim, 2015). Bakes et al. (2016) used 0.9\% for initial bolus and then $0.675 \%$ sodium chloride for maintenance. Research completed by Kuppermann et al. (2018), the PECARN DKA fluid study, reported that there was little difference in complications such as cerebral edema when comparing use of $0.9 \%$ and $0.45 \%$ saline. Shafi and Kumar (2018) postulated that use of a hypertonic solution of 3\% saline would decrease the likelihood of cerebral edema as it is the recommended treatment for cerebral edema; though it was found to be equivalent for rehydration, there was no decrease in incidence of cerebral edema but an increase in complications such as hypernatremia, hyperchloremia, and hyperchloremic 
metabolic acidosis. Santi et al. (2015) recommend the balanced salt solutions in any pediatric fluid resuscitation. Yung, Letton, and Keeley (2017) reported that though the balanced salt solution Hartman's solution was equivalent to $0.9 \%$ saline for correcting plasma bicarbonate, acid-base disturbances were corrected in less time with Hartman's solution. Bakes et al. (2016) found that a higher volume of fluid corrected the dehydration and acidosis, but the incidence of cerebral edema was higher in the patients who received more fluid in the first 8 hours of treatment. Kuppermann et al (2018) found that there were fewer clinical signs of brain injury and reduced mental status changes in the rapid rehydration group of their study, though the difference between the groups was not significant.

Guidelines and research agree that the fluid bolus should be given before the insulin is started. Two of the articles state that IV insulin should be started at $0.05-0.1 \mathrm{units} / \mathrm{kg} / \mathrm{hour}$ (Peeters et al., 2015), (Wolfsdorf et al., 2018) while others favor 0.05 units/kg/hour (Nallasamy, Jayashree, Singhi, \& Bansal, 2014) or 0.1 units/kg/hour (Kim, 2015), (Kuppermann et al., 2018), (Skitch \&Valani, 2015). Some did not list a preference (Nemri et al., 2016). Some articles validate the use of subcutaneous insulin in place of IV insulin (Cohen et al., 2017), (Razavi, Maher, \& Fredmal, 2018), while others accept subcutaneous insulin as a substitute when needed (Wolfsdorf et al., 2018). Shankar, Haque, Churchwell, and Russell (2007) validate the safety of beginning the long-acting subcutaneous insulin more than 4 hours before stopping IV-infused insulin. Harrison, Rustico, Palladino, Ferrara, and Hawkes (2017) reported that glargine insulin administered more than 4 hours before stopping intravenous insulin does not significantly increase the rate of hypoglycemia. Barski, Brandstaetter, Sagy, and Jotkowitz (2018) reported that co-administering long-acting insulin and intravenous insulin early in DKA treatment is well tolerated by patients and may decrease the amount of intravenous insulin needed, decrease the 
time required to correct the acidosis, and shorten hospitalization.

Monitoring is mostly congruent through the studies (Wolfsdorf, 2018), (Kim, 2015), (Koves et al., 2014), (Long \& Koyfman, 2017), (Peeters et al., 2018), (Skitch \&Valani, 2015), (von Oettingen, Rhodes, \& Wolfsdorf, 2018). All guidelines recommend hourly monitoring of vital signs and neurovascular checks due to the high morbidity and mortality rate of cerebral edema if not recognized and treated promptly. Hourly monitoring of capillary glucose levels is also advised (Wolfsdorf et al., 2018), (Kim, 2015), (Koves et al., 2014), (Long \& Koyfman, 2017), (Peeters et al., 2018), (Skitch \&Valani, 2015), and (von Oettingen et al., 2018). Most also recommend monitoring of electrolyte and venous blood gases every 2 to 4 hours to monitor efficacy of treatment and detect complications early. However, von Oettingen et al. (2018) reported that monitoring of electrolytes, bicarbonate, and anion gap were as effective as monitoring a blood gas for $\mathrm{pH}$. Wolfsdorf et al (2018) also recommends checking beta hydroxybutyrate at the beginning and throughout treatment if readily available to help differentiate between DKA and hyperchloremic metabolic acidosis as DKA corrects. This is because the treatment for hyperchloremic metabolic acidosis would be to decrease chlorine intake by stopping sodium chloride infusions or switching to a solution with lower or no sodium chloride, whereas the treatment of the DKA would be to continue the fluids (Wolfsdorf et al., 2018).

Hypokalemia is a common complication during DKA treatment for several reasons. Initially, intracellular potassium levels are depleted due to transcellular shifts caused by increased plasma osmolality that causes water and potassium to be pulled from the cells (Wolfsdorf et al., 2018). Some potassium is lost through vomiting and osmotic diuresis (Wolfsdorf et al., 2018). The depleted volume also triggers hyperaldosteronism, which promotes 
further potassium excretion (Wolfsdorf et al., 2018). Patients may present with elevated, normal, or reduced serum potassium; renal dysfunction resulting from hyperglycemia may contribute to hyperkalemia (Wolfsdorf et al., 2018). Once insulin is started and the acidosis begins to correct, potassium is pulled intracellularly, causing hypokalemia (Wolfsdorf et al., 2018). To correct for this, several of the guidelines and studies encourage potassium replacement early or even the incorporation of 20 to 40 milli-equivalents of potassium chloride or potassium phosphate (or a combination of the two), into the maintenance fluids (Kim, 2015), (Skitch \&Valani, 2015), and (Wolfsdorf et al., 2018).

In DKA hyperglycemia corrects before the acidosis. Therefore, to reduce the incidence of hypoglycemia throughout treatment, several articles encourage the addition of dextrose into the maintenance fluid once the patient's capillary blood glucose reaches less than $300 \mathrm{mg} / \mathrm{dL}$ or if the glucose level decreases by more than $90 \mathrm{mg} / \mathrm{dL}$ (Skitch \&Valani, 2015), (Peeters et al., 2018), (Wolfsdorf et al., 2018). Dhochak, Jayashree, and Singhi (2018) compare a one-bag (maintenance fluid bag changed to meet patient dextrose needs) and two-bag system (one bag has no dextrose and the other has $12.5 \%$; infusion rate changes are made in each for desired dextrose concentration). They found that while patient outcomes were similar with regards to effects on blood glucose and complications, the two-bag system required fewer fluid bag changes when patient dextrose needs changed (Dhochak et al., 2018).

Evidence shows that following a DKA protocol has several benefits. Some articles expressed how following a protocol improves patient outcomes such as shortening the length of hospitalization (Koves et al, 2014), (Horvat et al, 2018), and (Nemri et al, 2017) and decreasing incidence of cerebral edema when compared to not following a protocol (Koves et al., 2014). Another article by Skitch and Valani (2015) compared protocols implemented in 13 different 
hospitals in Canada and found several similarities in their DKA protocols that are comparable to recommendations made in other articles reviewed in this study. Peeters et al. (2015) described how DKA can be managed in a community hospital with a reliable protocol, close patient monitoring, and staff familiar with treating pediatric DKA or advised by an expert in DKA management. The findings of Peeters et al. (2015) are significant because generally pediatric patients experiencing DKA are transferred to a tertiary acute care setting to be managed by specialists. Nemri et al. (2017) reports reduced hospital stays when clinical practice guidelines are followed because standardizing care given to patients improves efficiency in administering treatment and identifying and addressing problems in earlier stages.

\section{Theoretical Framework}

Two theoretical models guided this project. The first and overarching theory was the Duffy Quality-Care Model as it is a guiding theory for nursing. Duffy and Hoskins (2003) through this model explained that providing quality nursing care improves patient outcomes. By exploring current clinical guidelines for the treatment of DKA and incorporating them into the current DKA protocol in place, the quality of nursing care, and the care provided by the healthcare team altogether, improved; thus, patient outcomes were, and will continue to be, optimized. By formatting the protocol in a simple-to-follow algorithm, including a table with maintenance fluids already calculated, and listing signs and symptoms of declining patient status, a lower margin for error was created. Staff, especially bedside nurses, were and will continue to be better equipped to identify complications early and implement corrective courses to prevent poor outcomes.

The theoretical model used to guide the implementation of this project was a five-stage model for the incorporation of current research and guidelines into practice called the Star Model 
of Knowledge Transformation (Stevens, 2012). (See Appendix A for model template). The first stage, or point, of the Star Model was discovery research, during which new knowledge was acquired through primary research (Stevens, 2012). Research studies performed for DKA issues, including various management options and the utilization of a protocol, qualify as discovery research. For this project, the primary research was not being performed by the project leaders, but available literature was being used to explore the findings of primary research studies on this topic.

The second point in the Star Model was evidence summary (Stevens, 2012). This stage was defined by the examination and compilation of the studies that have been completed (Stevens, 2012). This step has been carried out through the preceding literature review and critical appraisal of the DKA studies found in the literature, from which the information will be incorporated into the new protocol. A summary of the evidence was compiled in the literature synthesis.

The third point in the Star Model was the first of two parts of incorporating the new knowledge into actual practice (Stevens, 2012). In this stage, the synthetization of the available literature was organized into recommendations for practice (Stevens, 2012). This point was further operationalized with focus groups for assimilation of the guidelines and evidence from random control trials into the structure of the previous protocol to form the new DKA protocol.

The fourth point in the Star Model, and second in the two parts of incorporating the new knowledge, was practice integration (Stevens, 2012). During this step, the guidelines established in the previous point were integrated into a system for practice (Stevens, 2012). This was the step with which most healthcare providers are most familiar. For the project, this was the actual implementation of the protocol practice change, including the integration of the latest EBP 
guidelines into a new DKA protocol that was followed through an easier, algorithmic process and was incorporated into the EMR for application to practice.

The fifth point in the Star Model is process and outcome evaluation (Stevens, 2012). During this stage, both the results of the change and the process with which it was made are evaluated (Stevens, 2012). For this project, this point correlated with the evaluation of the project implementation. Faults and problems found with the process and outcomes of the change get evaluated as translational research, restarting the Star Model from the beginning in a cyclical fashion of incorporating evidence into practice. For the DKA project, process and outcome evaluation included a review of the change and implementation process for how this project was executed. Measurements included feasibility measures and staff satisfaction evaluations.

This framework offered a strong basis from which to incorporate a system change. It followed very naturally to the nursing process of assess, diagnose, research treatment, intervene, and evaluate but applied on a system level. The STAR model was an excellent guiding framework for this DKA project.

\section{Project}

\section{Congruence to Organization's Strategic Plan}

The mission statement of the tertiary care hospital through which this project was implemented was:

"To improve the health of West Virginians and all we serve through excellence in patient care, research, and education."

Further, the vision statement was as follows:

"To transform lives and eliminate health disparities through a nationally recognized patient-centered system of care that includes an expanded regional healthcare delivery 
system; consistent, integrated patient care recognized for delivering the right care in the right place at the right time at all sites; development of new approaches to improve healthcare, including team-based models of care, expanding WVU clinical and translational research; educational programs throughout the network recognized for training uniquely qualified healthcare team members and leaders; and a culture of performance and excellence throughout the network."

The underlying goal of this project was to improve the care that pediatric patients with DKA receive in a PICU in a tertiary care hospital in northern $\mathrm{WV}$, which was congruent with the points of expanding translational research and promoting a culture of excellence within the mission statement of the organization. This was accomplished through the integration of an evidence-based pediatric DKA treatment protocol into the regular workflow of the PICU.

\section{Evidence of Key Site Support}

One of the primary stakeholders is the chair of the hospital's Department of Pediatrics as well as a PICU attending pediatric intensivist. He agreed to serve as a content expert on the committee for this project. Another stakeholder, the nurse manager for the PICU, expressed support for this project and agreed to allot time during staff meetings to introduce this project to the staff. Verbal support from several of the bedside nurses on the PICU was acquired. A letter of support and approval from the research committee, led by another member of the project committee, can be found in Appendix B.

\section{Organizational and Project Needs Assessment}

A needs assessment was completed in the early stages of the project that identified the demand for this project change due to staff dissatisfaction with the adherence to the former DKA protocol and concern for the protocol being outdated with changing evidence-based guidelines, 
which could affect patient health outcomes. Needs identified include the demand for an updated, evidence-based protocol and then education of the staff for implementation of the protocol. The change project utilized the interventionist's time as well as the existing staff on the PICU. IT was utilized to incorporate the changes to the order set in the EMR. The optimized protocol expanded on the DKA protocol in place, so only minimal training to introduce the PICU staff to the changes was needed. Aside from the minimal training of personnel required to implement the new protocol, no additional time was added to workflow. The training was planned for 3 nonmandatory live online sessions lasting approximately 45 minutes, and through emails of the protocol.

\section{Identification of Project Resources}

Personnel. The stakeholders included: the change interventionist (a licensed family nurse practitioner with experience as a registered nurse in the PICU), pediatric intensivists, nurse practitioners (NP's), physician assistants (PA's), medical residents, and nursing staff of the PICU; pediatric endocrinologists; diabetes educators; information technology specialists who can make the needed changes within Epic and collect data from Epic; patients with DKA and their families. The primary interventionist undertook most of the change portion of the intervention through literature review, update of the protocol, request for implementation of the protocol into the EMR, education and support of staff during the implementation phase, and evaluation of staff satisfaction and utilization of the protocol. The pediatric intensivists, NP's, PA's, and medical residents provided feedback upon which the protocol was built and placed the orders in the electronic medical record, and then nurses evaluated and performed the interventions.

Materials. The materials required for this project included office supplies for copies of the protocol kept at the front desk and page protectors and 1-inch book rings for these copies, poster 
supplies for educational posters placed in the PICU, a promotional \$25 gift card, and the equipment necessary for the provision of patient care already allotted to the PICU.

Technology. Technology required to complete this project include the Epic EMR as well as information technology (IT) support for integration of changes made to the order set already existing in the EMR, platform for live online communication already allotted to the PICU, telephone equipment for communication regarding questions or concerns, and email availability for inter-unit communication.

\section{Strategic Analysis}

A strength, weaknesses, opportunities, threats (SWOT) analysis was performed for this project before implementation. Strengths identified include interventionist familiarity with the primary change site as the project is being led by a registered nurse (RN) with previous PICU experience as part of a doctoral nursing project, and existence of a DKA protocol in use (although this was not rigidly adhered to). There was also organizational support of the hospital (of the committee, from the PICU staff, and from the hospital's research committee) (See Appendix B for letter of site support), and the site was a Magnet-designated facility that supported nurse-led quality improvement projects.

One weakness identified was the potential that the change would not be accepted by the site since there was already a DKA protocol in use. There was a risk that not all staff would be committed to making this practice change since they may have preferred to avoid change to a newer protocol. Another possible weakness was that since the interventionist, the author of this paper, viewed this problem from the nursing role, the interventionist may have perceived the problem as larger than it was seen by the organization, and thus not receive the full support of the organization. 
One opportunity that existed was updating a protocol in use to match recently released guidelines. Another was updating staff knowledge about current guidelines and new literature that has been published within the last 5 years. This project was also an opportunity to educate newer staff about the pathophysiology of DKA and the rationale for each intervention. By refreshing staff knowledge about DKA treatment, staff was better equipped to critically think through outlying patient parameters to develop the most appropriate treatment plans.

One perceived threat was the staff resistance to making a change that is present with any practice change. There was the potential that the protocol may not be seen as useful since an effective protocol was already in place and therefore the possibility that the new protocol may not be utilized. Another threat was that the timeframe for the project would not be long enough to acquire adequate data to evaluate the effectiveness of the intervention. Though this PICU treats pediatric patients with DKA from WV and from surrounding areas, patients experiencing DKA are admitted on a sporadic basis.

\section{Feasibility Analysis}

The project intervention was intended to incorporate current, well-referenced clinical practice guidelines into regular practice in the PICU in a format that was efficient and easy to use. The optimized protocol was built upon the fundamentals of the previous protocol in use in the PICU, so training was built on the current protocol and highlighted the EBP recommendations integrated into the new protocol. This training was completed through live online meetings and poster presentation. The changes made were within the limits of the abilities and scopes of practice for the staff affected, primarily physicians, residents, nurse practitioners, physician assistants, and registered nurses. No additional staffing was required to make these changes. There were no confidentiality, privacy, or security issues with this change project. The 
functional requirements involved updating an existing order set and communicating primarily via email and live online meetings.

\section{Market Analysis}

According to Desai et al. (2018), an approximate total aggregate cost for DKA hospitalizations in the United States in 2014 was $\$ 5.1$ billion dollars. Desai et al. (2018) reported that each admission cost approximately $\$ 26,566$. Horvat et al. (2018) reported that an estimated $\$ 90$ million per year are spent to treat DKA in pediatric and adolescent patients in the United States. The PICU site of the project had 113 pediatric DKA patient encounters for 2017 and 74 in 2018 (Epic, 2019). Therefore, based on the 2014 admission rate quoted by Desai et al. (2018), between approximately $\$ 1,965,884$ and $\$ 3,001,958$ has been spent for DKA admissions to the PICU in a single year. Complications in the disease process and mismanagement of treatment can worsen patient outcomes and therefore increase the need for resources, both financial and personnel, by increasing length of stay and number of interventions necessary to treat the patient. Therefore, it was imperative that the PICU implement an optimized DKA protocol that was supported by evidence, that complied with clinical guidelines, and that will be followed by the staff.

Aside from the resource savings, there were other benefits to the organization, the patients, and the staff from this project. Naturally, patients benefitted from health outcomes that come with better healthcare and reduced length of stay. Both the staff and the organization benefitted from endorsing a nurse-led research project within a Magnet ${ }^{\circledR}$ hospital. By endorsing a nurse-led research project, staff satisfaction was increased as nurses were empowered to make positive changes in their working environments that benefitted both patients and staff. The organization also could potentially gain the recognition that comes with promoting nurse-led 
projects that enhance patient outcomes.

\section{Sales/Marketing}

Marketing for this project included posters of the optimized DKA protocol in high-traffic areas, hard copies of the protocol kept at the front desk to be viewed at any time, and live online education sessions. It is the intention that in the future copies of this new protocol will be dispersed to and used by other healthcare facilities in West Virginia. In the future healthcare providers throughout West Virginia may use these same protocols to initiate care before transfer or possibly even treat milder cases of DKA within their own facilities, allowing WV children with DKA to be treated in their hometowns.

\section{Operations and Financial Plan}

The cost to the organization was minimal because it was a project being performed by a doctoral nursing student who agreed to undertake the project without salary reimbursement, which if performed by an outside agency would have been approximately $\$ 2,900$. All staff education took place online without mandatory participation with approximately 30 nurses participating in the live educational sessions who were compensated by the organization for 45 minutes of time outside of their scheduled time, approximately $\$ 650$ total. Expenses for marketing, educational materials, and project supplies were paid for by the change agent as they included poster supplies and office supplies needed to print copies of the protocol to place at the PICU front desk. This cost was $\$ 72.11$. Much of the education was completed with online education sessions, which utilized a program already purchased by the institution for other purposes and therefore did not incur additional cost to the institution. The change interventionist had budgeted for $\$ 100$ for hospitalities to allow for snacks at meetings to encourage engagement, but due to a global pandemic prohibiting the change agent from visiting the site for education, 
this planned fund was not used. However, \$25 of this budget was used to purchase a gift card as incentive for staff to complete the post-implementation survey. No significant travel expenses were sustained since the change interventionist was within 5 miles of the change site and because the site prohibited live visitation due to global pandemic during the time the project was implemented. There were no costs to the organization for the change made to the EMR since the changes were made by the organization's information technology department rather than requiring the assistance of an EMR consultant (See Appendix C for budget).

\section{Description and Design}

According to Koves et al. (2014), implementing an evidence-based pathway for DKA treatment in pediatric patients improves the level of care patients receive and ultimately improves outcomes. While a DKA protocol was already in place in the PICU of this tertiary care hospital in northern WV, it was lacking the evidence base and optimized pathway. The design for this practice change was to optimize the protocol pathway to comply with current evidencebased guidelines presented by Wolfsdorf et al. (2018). The protocol was formulated into an algorithm, calculation chart, and helpful information for physicians and nurses. The algorithm was created using a combination of resources from the ISPAD Clinical Practice Consensus Guidelines 2018: Diabetic ketoacidosis and hyperglycemic hyperosmolar state (Wolfsdorf et al., 2018) (see Appendix D1) and the former DKA protocol previously used in the PICU (see Appendix D2). Accompanying the algorithm was a quick reference calculation chart for fluid maintenance and replacement volumes (see Appendix D3). This calculation was based on a fluid calculation for 1.5 times maintenance, congruent with guidelines at the time the project was developed, and the calculation used by the former protocol. Continuation of use of this calculation helped improve likelihood of acceptance of the protocol. A copy of the new protocol 
implemented is available in Appendix D4.

This practice change project was designed for the clinicians and staff working in the PICU and caring for the pediatric patients experiencing DKA. The staff at this PICU were already following a paper DKA protocol for more than a decade with an associated order set in the EMR. After completing the literature review and integrating the evidence-based guidelines with the former protocol to develop the new product, clinician feedback was sought. A focus group was held with the physician staff before the protocol revision to further develop the protocol with clinician input. Informational sessions were held with various staff via live online meetings to introduce the new protocol and address questions and concerns. There were two sessions for the nursing staff and one session for the medical residents and interns. These sessions lasted approximately 45 minutes and included education pertaining to the pathophysiology of DKA, an introduction to the new protocol, and rationale for the steps in the protocol. Additional educational sessions were offered to the advanced practice provider staff and physicians as well before implementation of the revised protocol but were declined in favor of a copy of the new protocol and the opportunity to email questions and concerns to the author of this project. Also, informational posters were placed in main areas of the PICU and in the resident charting room prior to the implementation of the project to introduce the protocol to as many people as possible before it was implemented.

To further promote use of the protocol, paper copies of the new protocol were available for all staff, and an electronic copy was available through the unit web-based collaborative platform SharePoint. A staff survey was collected for process and outcome evaluation before implementation and after the protocol was in use for 3 months (see Appendix E for Staff Survey). 


\section{Measurable Project Objectives}

The objectives of this project were designed using specific, measurable, achievable, realistic, and timely (SMART) goals (Corporate Finance Institute, 2015).

Objective \#1: An evidence-based protocol for managing pediatric patients in DKA according to current guidelines would be used in the PICU by the beginning of May 2020.

Outcome 1A: A review of the current literature to incorporate evidence-based practice recommendations would be completed.

Outcome 1B: A streamlined protocol in a simple-to-read algorithm that was compliant with current guidelines would be created.

The impetus for this objective and outcomes was to allow providers to have access to evidencebased practice in a convenient source that was easy to use. The plan for evaluating this outcome was to compare the updated protocol to the clinical guidelines put forth by the organizations cited in Wolfsdorf et al. (2018).

Objective \#2: An assessment of the feasibility of long-term integration and sustainability of the revised DKA protocol into practice in the PICU would be completed by the end of the project.

Outcome 2A: The revised DKA protocol is accepted by the staff.

Outcome 2B: The revised protocol is adaptable to new unit circumstances and guideline updates.

The plan for evaluation of Outcome 2A was through surveys that included both Likert scale questions and open-ended questions that was implemented in the beginning and at the end of the pilot implementation (See Appendix E for Staff Survey). Since these surveys were developed by 
the interventionist, the validity and reliability were not verified. Additional evaluation was planned through observational and anecdotal evidence collected by the content expert assisting the interventionist with this project. Evaluation of Outcome $2 \mathrm{~B}$ was planned by making changes to the protocol recommended by the pediatric endocrinologist early in the implementation stage and planning changes for improvements to the protocol at the end of the pilot implementation.

\section{Timeline of Project}

The initial project idea and literature review were initiated in May 2019, and subsequent progress was tracked from that time (See Appendices F1 and F2 for detailed Project Timeline). In summary, specific milestones included:

- May 2019: The engagement of stakeholders and drafting of the DKA protocol.

- June 2019: Preparation of educational tools and evaluation surveys.

- May-July 2019: Focus groups to engage stakeholders.

- December 2019: The proposal was provided to project committee members for review and approval.

- March 2020: Proposal was provided to the Nursing Research Committee for review and deemed approved.

- April 2020: Proposal submitted to the IRB and deemed approved.

- April-October 2020: Detailed revisions of the new protocol in the PICU before implementation were requested by the chief attending physician of the PICU; these revisions had to be discussed by all stakeholders and several revisions occurred over time before the protocol was approved by all staff. November 2020-January 2021: Repeated revisions were made to the staff educational PowerPoint presentation, including adding 
more education about pathophysiology in the protocol education presentation.

- February 2021: The 3 live online educational sessions were completed. The initial staff satisfaction survey link was delivered by email to the staff after the educational sessions were complete.

- March 1, 2021-May 31, 2021: The new DKA protocol was implemented in the PICU. Troubleshooting and rapid cycle quality improvement measures were provided as needed.

- June 2021: The final staff satisfaction survey link was sent to staff, and data analysis was performed.

\section{Results}

Objective 1: This first objective was partially met. Though the 2 expected outcomes for this objective were met, they were not met by the May 2020 timeline.

Outcome 1A: The literature review made known the current body of evidence-based practice to incorporate into the protocol, and updates were made within the protocol as appropriate.

Outcome 1B: A streamlined algorithmic protocol was created through the use of evidence-based guidelines and stakeholder engagement in focus groups. The protocol was revised as a flowsheet algorithm with a calculation sheet attached to reduce calculation errors at the advice of the content expert on the change agent's committee. In addition, there was a page of helpful information for physicians and another page of helpful information for nurses to reference. There was also a reference for identifying and treating cerebral edema at the top of the first page of the protocol at the advice of one of the attending physicians in the PICU.

Objective 2: The second outcome of ascertaining feasibility of long-term integration and 
sustainability of the revised DKA protocol into practice in PICU was met. According to Bowen et al (2009), feasibility of implementing and sustaining an intervention or practice change can be measured through acceptability, demand, implementation, practicality, adaptation, integration, expansion, and limited-efficacy testing.

Outcome 2A: Acceptability was shown to be met in that $78.9 \%$ of staff members who took the survey at the end of the 3-month trial said they were extremely likely or somewhat likely to use the new protocol. Implementation was achieved in that the protocol was fully implemented into use on the PICU between March 1, 2021 and May 31,2021 and continued to be in use at the time this paper was written. The ability to integrate the protocol into practice further demonstrates that the protocol was acceptable to the staff. Integration occurred through the implementation of the revised protocol during the pilot trial.

Outcome 2B: The protocol has been shown to be practical and adaptable. Practicality was demonstrated in that the revised protocol was adopted into practice with no need for new equipment or increase of time commitment on the part of the staff caring for patients with DKA. Adaptation was displayed early in the implementation when the pediatric endocrinologist recommended changes to initial labs collected as well as ways to identify high-risk patients. The protocol will continue to be evaluated for the need to make revisions, further demonstrating its adaptability.

Feasibility was further confirmed based on the measurement criteria laid out by Bowen et al. (2009) through limited-efficacy testing and expansion. Limited-efficacy testing was achieved through the implementation of this pilot study. Though it has not transpired at the completion of this project, expansion could potentially occur in the future through dissemination of the protocol 
to other hospitals throughout West Virginia within the healthcare system of this hospital. Once revisions are made to the satisfaction of the PICU team, it is likely that copies of the protocol will be dispersed to other hospital emergency departments when the transport team travels to transport patients. A staff survey was devised to measure some of these elements of feasibility and sustainability.

\section{Data Analysis}

The surveys were offered to the staff at the beginning and end of implementation (See Appendix E for Staff Survey). The initial survey was completed by 18 staff members and the final by 20 staff members out of approximately 130-150 possible staff that included physicians, physician assistants, nurse practitioners, nurses, pharmacy staff, and medical students/residents. Of note, there were 21 entries in the final survey, but one survey taker only completed the demographics questions and did not complete any questions pertaining to satisfaction of the protocols. Therefore, this person's survey answers were disregarded in the final analysis. (See Appendix G: Survey Results Graphs to see graphs of answers to each multiple choice survey question.)

Questions 1 and 2 of the staff survey were demographic questions to help assess the diversity of the sample who completed the survey. Question 1 showed most of the survey-takers were nurses. This was logical because most staff members in the PICU were nurses. Question 2 demonstrated that there were more experienced staff members answering the pre-implementation survey with 3 people in each tier except the tier of "10 years or greater", which was answered 9 people. There was a relatively equal distribution of experience of staff members who took the post-implementation survey, with 4 to 7 people at each tier of experience.

Question 3 established that $100 \%$ of both samples were familiar with the previous DKA 
protocol. Question 4 was used to assess the survey taker's familiarity with the revisions made to establish the new protocol. Because the options were not completely the same between the preimplementation and post-implementation surveys, no t-test was performed on this question.

Question 5 asked about perceived compliance to the previous protocol and then the new protocol. The numeric mean of the pre-implementation protocol was 2.22 , which translated to the average perception of compliance with the previous protocol was "somewhat well". The numeric mean of the post-implementation protocol was 2.1 , so the staff that completed the postimplementation survey also thought that the previous protocol was followed "somewhat well". A t-test was completed for this, and $\mathrm{p}=0.348$. Therefore, the null hypothesis was confirmed, and there was no statistical significance between the answer to the question concerning the previous protocol from the pre-implementation to the post-implementation. However, the numeric means varied regarding staff perception of adherence of the new protocol from the pre-implementation to the post-implementation. The numeric mean of adherence to the new protocol on the preimplementation survey was 5.278, which translated to "not well at all", but on the postimplementation survey it was 3.3 , which translated to "moderately well". In this case, $p=0.001$, which confirmed statistical significance in pre- and post-implementation means. However, a strong confounding factor to consider was that in the pre-implementation survey most survey takers answers "unsure" as expected since they had not used the protocol up to that point, which translated numerically to a 7 in the data set. When averaged, this greatly increased the mean. Fewer survey takers answered "unsure" or "have not had a chance to use yet" in the postimplementation survey, which created a higher mean value.

Question 6 asked the survey takers to rate the ease of use of both the previous DKA protocol and the new DKA protocol. For the previous DKA protocol, the numeric mean of this 
question on the pre-implementation survey was 1.944 , which translated to "very easy" and the post-implementation survey was 1.9 . For this part of the question, $\mathrm{p}=0.445$ and therefore was not statistically significant. For the new DKA protocol, the numeric mean for this question on the pre-implementation survey was 3.22, which translated to "neither easy nor complicated", and the post-implementation survey was 3.15 . For this part of the question, $p=0.45$ and therefore was not statistically significant. Again, for this question it must be considered that there were more people answering "did not use" in the pre-implementation survey than the post-implementation survey.

Question 7 asked the survey takers how likely they were to use the new protocol. The mean of the results for the pre-implementation survey was 1.278 , which translated to "extremely likely". The mean of the results for this question on the post-implementation survey was 1.895 , which rounded more closely to "somewhat likely." For this question, the $p=0.05$, which was statistically insignificant.

Question 8 asked the survey takers how well the new protocol followed their expected treatment plan for DKA. The mean of the results for the pre-implementation survey was 1.5 , which was halfway between "extremely well" and "very well". The mean of the results for the post-implementation survey was 2.526 , which was halfway between "very well” and "moderately well". The $\mathrm{p}=0.002$, which indicated a statistically significant change from the first survey to the second.

Questions 9 through 13 were open-ended questions. Question 9 asked the staff something they liked about the previous DKA protocol. Themes displayed in the pre-implementation survey were ease of use, precision, everything, nothing, and unsure. Themes discovered in the postimplementation survey were allowance for autonomy, effectiveness, ease of use, adaptability, 
and familiarity.

Question 10 requested staff recommendations for changes they thought should be made to the previous protocol. Themes that appeared for this question in the pre-implementation survey were format, compliance to evidence-based practices, universal use by staff, and no changes. Themes that appeared in the post-implementation survey were compliance to evidencebased practices, format, universal use by staff, and no changes.

Question 11 asked the staff to list something they liked about the new protocol. Themes found in the pre-implementation survey were format, evidence-based, and unsure. Themes displayed in the post-implementation survey were format, evidence-based, and nothing.

Like question 10, question 12 inquired about changes the survey takers thought should be made to the new protocol. Everyone who answered this question on the pre-implementation survey wrote that no changes needed to be made. Themes discovered on the post-implementation survey were that it does not correct for increasing glucose levels, that there was too much titration, that it was not specific enough, and that it was not universally followed by staff. Other responses included a suggestion to change the first treatment for cerebral edema from mannitol to $3 \%$ saline. Another person wrote that they did not feel familiar enough with the new protocol to make suggestions.

Question 13 asked the staff whether they preferred the old or new protocol and why. In the pre-implementation survey, there was an even distribution between those who preferred the new protocol and those who were not sure which they preferred. There was one person who said they preferred the old. Reasons given for preference for the new protocol were ease of use, readability, more detailed, more user-friendly, recent research that includes evidence-based practice. People who chose unsure primarily stated that they wanted to use the protocol before 
they decided or that they thought both the old and new protocols seemed easy to use and effective. In the post-implementation survey, there were 8 staff members who preferred the new protocol and 11 staff members who preferred the old protocol. Reasons listed for preference of the old protocol were allowance for clinical judgment and autonomy, more precise than new protocol, easier to follow, effective, patients correct quicker with old protocol, and has been in use longer so more comfortable to teach to new staff. Those who preferred the new protocol listed their reasons as the new protocol was easier to follow due to clearer instructions, the flowchart, and the calculations chart (See Appendix H: Themes of Open-Ended Questions of Surveys).

Something else was revealed by the staff survey. Prolonged elevated glucose levels were identified by staff during the implementation phase. One possible solution is adding the logic question into the algorithm for managing a patient whose finger stick glucose levels start rising instead of decreasing.

\section{Discussion}

As with any trial, unexpected circumstances arose. One was the delay in implementation of the pilot trial due in part to the global pandemic. Managing the pandemic surpassed the DKA protocol revision and implementation in priority to all stakeholders involved. This caused a delay in the revision and approval process of the DKA protocol before implementation in the PICU. The pandemic forced creativity in finding ways to communicate with and educate staff since the interventionist was not permitted into the hospital for in-person meetings or educations sessions. All communication was performed through email and live online meetings. Second was the delay in receiving feedback from stakeholders. Once the protocol was implemented in the PICU, the change agent was unable to visit the site to address questions or problems that were found after 
the implementation, though contact information was provided and a unit champion appointed. This potentially impacted the overall acceptance of the new protocol in that the staff may have believed that the issues not discovered until implementation would be left in the protocol beyond the pilot trial.

After implementation of the pilot phase of the project had started, the pediatric endocrinologist recommended modifications to the protocol that had not been suggested during the development stage. Another unintended consequence of the actual implementation of the protocol was prolonged periods of elevated glucose levels in the patients. However, after evaluating the protocol, a potential cause was found that a step for correcting glucose that increased above $300 \mathrm{mg} / \mathrm{dl}$ after incorporating dextrose into the maintenance fluid was missed in the maintenance IV fluid titration algorithm. Modification of the algorithm may correct this issue in the future, but this change to the protocol is under deliberation by the PICU team. This will be accomplished through further collaboration with the PICU team.

Despite these obstacles, the overarching goal of providing an evidence-based protocol that was easy to use was evident in that the calculation sheet and flow of the protocol were frequently listed as items the survey takers liked about the revised protocol in the final staff survey (See Appendix H for Themes of Open-Ended Questions of the Surveys).

Limitations. As was mentioned in the Results section, there were some elements of the survey to be considered when evaluating the results. First, unpaired t tests were performed on the survey because there were no tracking elements to see how many, if any, of the same staff completed both the pre- and post-implementation surveys. Though the survey was offered to nearly 150 people, only 18 to 20 people completed each survey. This was a significantly small sample size and may not have captured the sentiments of most of the staff. In addition, the pre- 
implementation survey did not reach the pharmacy staff though the post-implementation survey did. The rest of the staff had access to both surveys. Further, it was learned after implementation that the pharmacy staff never used the old or new protocols but had their own protocol that centered on preparing the insulin and maintenance fluid bags for the protocols. Since both protocols were using the same concentration of insulin and the same maintenance fluids, the pharmacy staff would not have seen a difference in protocols during the implementation trial.

Attempts were made to collect data from the EMR as another measure of adherence to the protocol during the pilot trial with the plan to compare the percentage of patients for whom the lab beta-hydroxybutyrate was ordered during the pilot to the percentage of patients for whom the same lab was ordered during the previous year. The rationale for evaluating the use of betahydroxybutyrate was that this lab order was not a part of the previous protocol but was added into the revision. Therefore, if beta-hydroxybutyrate was ordered consistently during the pilot, this would have indicated adherence to the new protocol. However, interrogation of the EMR was fraught with obstacles, requiring interventionist access to patient charts for research purposes that was not approved by the organization during the timeframe of the data analysis period. Members of the PICU team will continue this work and analysts for the organization are working on evaluating this data.

Similarly, another data point that would have been helpful to assess was how often Lantus was administered on the first night of admission. Though this was beginning to become regular practice per the endocrinologists' request, administration of Lantus during the first night of admission was not part of the original protocol but was implemented into the revision. At the time of conclusion of the DNP project, the PICU team and analysts were working to acquire this data. 
Strengths. The two theoretical frameworks that supported this project were the Duffy QualityCare Model and the Star Model of Knowledge Transformation. This project provided tools to help improve the quality of the care nurses provided to pediatric patients with DKA. The protocol provided a list of symptoms to more quickly identify if patients were experiencing cerebral edema and the steps to take if cerebral edema was suspected. The protocol also provided a key for identifying the appropriate amount of maintenance fluid a patient receives per hour based on weight. Further, the steps were laid out in a simple, algorithmic way to help best deliver the highest quality of care.

The steps of the Star Model of Knowledge Transformation led the progress of this project. Discovery research completed in previous projects was researched and appraised before proceeding to the second stage of evidence summary and the third stage of translation to guidelines. The necessary changes were made to the previous protocol to concur with what was found in the research. The fourth stage of practice integration took place during the pilot study. Finally, the process outcome was evaluated through the staff surveys and an interview with the content expert of the committee who used the revised protocol. Information was acquired through the surveys and interview to discover new information about the revised protocol for improvements that can be made. As in the theory, the cycle of learning and reforming will continue beyond the range of this project.

Despite the mix of staff member preference for protocols, it is likely that this revised DKA protocol will remain in practice in the PICU. Staff members seem to appreciate the use of the flowchart and algorithm to organize the steps of managing a patient with DKA more easily. They also seem to appreciate the page of maintenance fluid calculations.

\section{Recommendations}


It is the recommendation of this change agent that a small interdisciplinary committee of staff members evaluate the protocol periodically, at least every 5 years, to evaluate the protocol's compliance to current guidelines and make updates as appropriate. It is the further hope of this change agent that another DNP student would expand this project to disseminate the protocol to other hospitals in West Virginia, or at least hospitals within this hospital's health system. If unable to disseminate this protocol, this change agent would like to see imparted to hospitals in West Virginia the importance of implementing an evidence-based protocol for managing care of pediatric patients with DKA.

Some improvements will need to be made to improve staff satisfaction. Foremost is assessing and addressing the issue of no step for managing glucoses that increase above 300 $\mathrm{mg} / \mathrm{dl}$ after integrating dextrose into the maintenance fluid. At this time, it is recommended that this issue be evaluated further to see if this decreases incidence of hypoglycemia while correcting acidosis.

Another change is substituting a different maintenance fluid for normal saline to help decrease the incidence of hyperchloremic acidosis. This was a change some of the attending physicians requested to make during the revision stage of the protocol but was not possible at the time of the project due to the unknown compatibility of the maintenance fluid they wanted to use (Plasmalyte) with insulin. As people continue to use the protocol, more situations will likely occur that require revisions to the protocol. It was the intention that the protocol would be simple to edit over time when problems were discovered or new information revealed through research.

\section{Attainment of DNP Essentials}

As a DNP project, it is vital that this project aligns with all eight of the DNP Essentials. The first essential, the scientific underpinnings of practice, was met through the study of the 
mechanisms that cause DKA. Gaining a strong understanding of how interventions

recommended in nationally and internationally accepted guidelines affect these mechanisms was vital as well. Further, under the title of scientific underpinnings, it was imperative for the DNP to understand possible side effects each treatment could cause and be prepared to manage those side effects as well.

The second essential, organizational and systems leadership for quality improvement, was met through the steps required to accomplish a quality improvement project within a health system. The DNP student as a change agent operated as a leader to develop and advance the project within the PICU so that its staff had access to an updated, evidence-based protocol for reference when treating pediatric patients with DKA. The DNP student gained an understanding of system procedures and protocols for making quality improvement changes and successfully navigating said protocols.

Clinical scholarship and analytical methods for evidence-based practice, the third essential, was met through the literature synthesis and review. During this process, an unchanged protocol in place for several years was being evaluated in comparison to current EBP guidelines. It was important that the DNP follow guidelines approved by experts in the field, but it was also important to consider the shareholders affected by the change. The DNP knew that often the best new protocol to implement was one that, though complying with current guidelines, also held similarities to the previous protocol. The DNP student must also be able to gather and analyze data throughout the project and identify patterns and trends. In addition, analyzing the results of the protocol and surveys from the staff and using knowledge acquired to critically think through and identify causes for problems and propose solutions to those problems fell under this category as well. 
The fourth essential, information systems and technology and patient care technology for improvement and transformation of health care, was met through incorporating various forms of information technology to disseminate the evidence-based protocol to all staff using the protocol. This was accomplished by disseminating the revised protocol through the web-based collaborative platform SharePoint. The fourth essential was also met by using WebEx for live educational meetings for the staff. Also, this essential was met by using email to communicate with the staff for the purposes of revising the protocol, planning various stages in the process of preparing and implementing the protocol, addressing questions and concerns as the protocol was put into use, and dispersing surveys for evaluating the project. The surveys for evaluating this project were completed and evaluated through the internet program Qualtrics.

The fifth essential of health care policy for advocacy in health care was met through the decision to compare a protocol that had been used for years to current guidelines and promote best practice policies to positively impact patient outcomes. By implementing a new protocol that complied with current guidelines, a health care policy was changed to advocate for the best evidence-based practice to be used when caring for pediatric patients with DKA. Incorporating current EBP into routine practice promoted the best outcomes for patients, epitomizing advocacy in health care. In addition, observing that something in the protocol was not working and collaborating with others to make improvements also demonstrated advocacy in healthcare.

The sixth essential of inter-professional collaboration for improving patient and population health outcomes was met through cooperating with experts in various fields, including pediatric endocrinologists, pediatric intensivists, nurse practitioners, physician assistants, bedside nurses, diabetes educators, pharmacists, IT specialists, data analysts, system administrators, and research committees. This inter-professional team had the common goal of 
providing a DKA protocol that complied with national and international guidelines for DKA treatment that was simple to follow. Each member of the team brought a different perspective to consider when devising the protocol, enriching the quality of the final product.

The seventh essential of clinical prevention and population health for improving the nation's health was met in various ways. First, this essential was met in the preparation phase of the project by interviewing children and adolescents with diabetes during their yearly camp to gain a better understanding of their experiences while hospitalized. This also allowed an opportunity to explain to the children and adolescents the rationale for practices and protocols in the hospital. Second, the seventh essential was met by implementing a DKA protocol that could potentially decrease the risk for morbidities and mortalities associated with poor management of DKA in pediatric patients. Though the intervention did not prevent hospitalizations, it could decrease the occurrences of complications so patients admitted with DKA could recover from DKA more quickly and be discharged from the hospital sooner, possibly decreasing nosocomial infections in a population more susceptible to infection. Third, the change agent presented this project in various conferences to disseminate information about the importance of implementing a protocol for the management of DKA in the pediatric population.

Finally, the eighth essential of advanced nursing practice was met through the application of the nursing process to address the quality improvement change. First the system was assessed through focus groups, anecdotal evidence, data collection, and literature review. The diagnosis was made that a poorly referenced DKA protocol that had been deemed long and difficult to follow had been used without EBP updates for over 10 years and modernizing it was a quality improvement measure that could promote better outcomes for pediatric patients with DKA. Plans were made for the implementation of the new protocol. Once the plans were approved, the 
planned steps were taken to approve and implement the new protocol. Finally, the success of the implantation was evaluated to determine whether the protocol was accepted and used, and challenges that were identified were addressed.

\section{Summary}

DKA is a serious complication of diabetes, especially in pediatric patients, that can lead to debilitating conditions and even death if untreated or poorly managed. To prevent morbidity and mortality in pediatric patients, it is important that the PICU at a tertiary care center in northern West Virginia have an up-to-date, evidence-based DKA protocol in place. This protocol was updated to comply with the published, peer-reviewed literature and formatted to be quickly and easily utilized by the PICU staff. Though this PICU had a DKA protocol in place, the protocol had not been updated to EBP for over a decade, and it was not consistently followed. The purpose of this DNP quality improvement project was to build upon the foundation of the original protocol an updated, easy-to-follow protocol algorithm that the staff would use to treat DKA. This DNP project aligned with the mission statement of the organization as well as the eight DNP Essentials. By updating the pediatric DKA protocol, the PICU will continue providing the highest quality, EBP-influenced care to the pediatric patients of WV and surrounding areas that experience DKA. 
Appendix A: Star Model of Knowledge Transformation

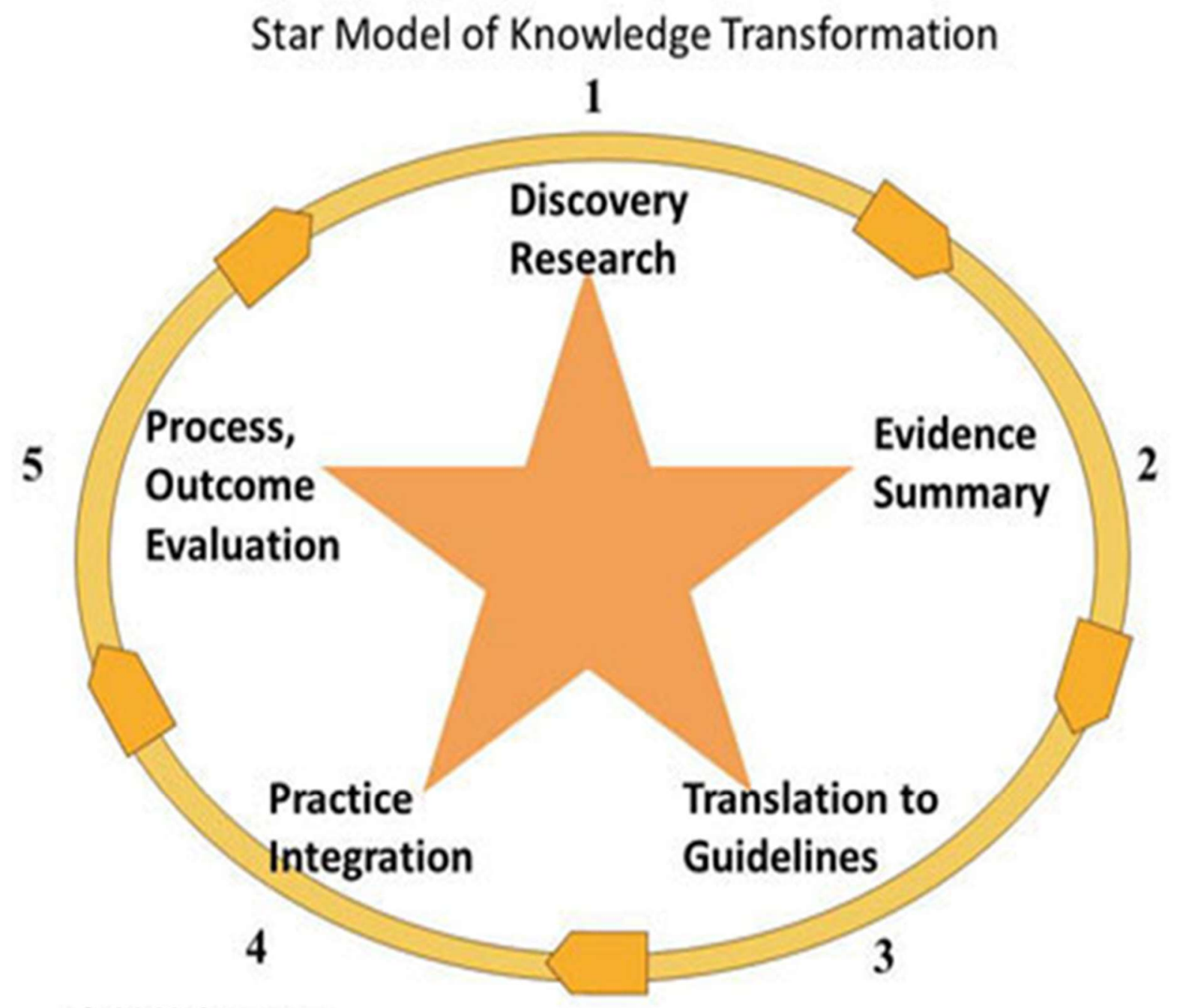

다 2012 Stevens 


\section{Appendix B: Organization Letter of Support}

March 30, 2020

Jennifer Burky, BSN, RN

WVU School of Nursing

Morgantown, WV 26506

To the WVU Institutional Review Board

The WVUH Research and Evidence-Based Practice Council supports the project undertaken by Jennifer Burky, "Optimizing a Pediatric DKA Protocol in a Tertiary Care Hospital System in Northem WV." This is a very important project as it has implications that will help to improve the care that patients receive and support the staff that care for them. All necessary resources will be provided to them as they undertake this project. The project outcomes will be used to revise/modify the program as necessary.

The Research and Evidence-Based Practice Council at WVUH grants you permission to complete your project with the following stipulations:

1) Permission is granted based on the project being carried out precisely as defined in your methodology

2) Permission is granted contingent upon approval and/or recommendations of the WVU Institutional Review Board

3) At the mid-point and at the completion of the study, you are requested to share your findings with the Nursing Research and Evidence-Based Council

Please forward me the WVU IRB approval letter for our files.

Best wishes to you in this endeavor!

Cordially,

Rya M. Streupe

Lya M. Stroupe DNP, APRN, CPNP, NEA-BC

Manager of Nursing Research and Professional Development/Magnet ${ }^{\circ}$ Program Director/Transition to Practice

Program Director

Nursing Administration/wvu Medicine

One Medical Center Drive /PO Box 8227

Morgantown, WV 26506-8227

304.293.1417 strounel@mumedicine.org 
Appendix C: Budget Plan

\begin{tabular}{|c|c|c|}
\hline Budget Categories & Personal Funds & Organizational Contributions \\
\hline ADMINISTRATIVE COSTS & $\$ 2900$ & $\$ 650$ \\
\hline $\begin{array}{l}\text { MARKETING, } \\
\text { EDUCATIONAL } \\
\text { MATERIALS, PROJECT } \\
\text { SUPPLIES (office supplies, } \\
\text { postage, printing, etc.) }\end{array}$ & $\$ 72.11$ & $\$ 0$ \\
\hline INCENTIVES & $\$ 25$ & $\$ 0$ \\
\hline $\begin{array}{l}\text { HOSPITALITY (food, room } \\
\text { rentals, etc.) (UNABLE TO } \\
\text { USE DUE TO GLOBAL } \\
\text { PANDEMIC) }\end{array}$ & $\$ 0$ & $\$ 0$ \\
\hline TRAVEL EXPENSES & $\$ 0$ & $\$ 0$ \\
\hline OTHER & $\$ 0$ & $\$ 0$ \\
\hline TOTALS & $\$ 2,997.11$ & $\$ 652.50$ \\
\hline
\end{tabular}




\section{Detailed Budget Description:}

1. Administrative Justification: $\sim 100$ hrs. of donated time by change agent $X \sim \$ 29 / \mathrm{hr} ; \sim 30$ RN's X $\$ 29$ \& providers getting $\sim 45$ min of education

2. Marketing, Educational Materials, and Project Supplies - \$72.11 for poster board, supplies, and lamination for DKA protocol posters hung in PICU; paper, ink, page protectors, and 1 -inch book rings for copies of protocol kept on unit.

3. Incentives Justification: $\$ 25$ gift for drawing to incentivize completing final survey (Note: names not associated with answers of surveys to maintain anonymity)

4. Hospitality Justification: Unable to use due to limitations set in response to the global pandemic

5. Travel Expenses Justification: No travel necessary as communication maintained through email and live online meetings.

7. Other Justification: Epic Implementation was free because completed by organization IT instead of agents from EMR company 


\section{Appendix D1 - ISPAD Clinical Practice Guidelines Algorithm 2018}

160 WI LEY WISPAD WOLFSORF ETAL.

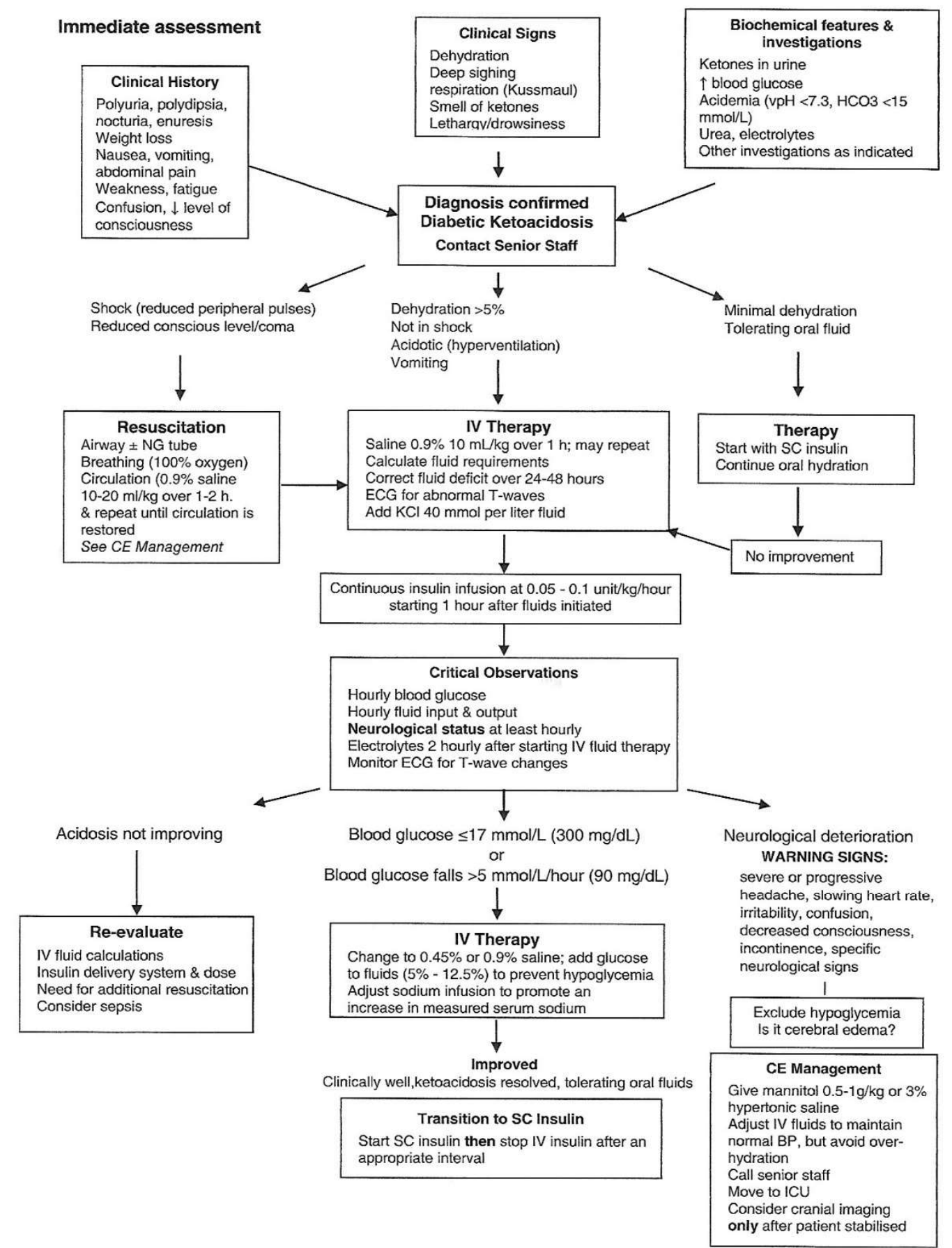

FIGURE 2 Algorithm for the management of diabetic ketoacidosis. Adapted from Pinhas-Hamiel and Sperling. ${ }^{271}$ NG, nasogastric; SC, subcutaneous 


\section{Appendix D2: Previous PICU DKA Protocol}

\section{WVU PICU DKA Guidelines}

Initial Labs:

Blood: Bedside glucose, glycosylated hemoglobin, lytes, $\mathrm{Ca}, \mathrm{Mg}, \mathrm{PO} 4, \mathrm{BUN}$, creatinine, glucose, VBG

Urine: bedside glucose \& ketones q void

Insulin:

Continuous low dose insulin infusion piggybacked into IV line to correct the acidosis and hyperglycemia. To reduce the risk of cerebral edema, delay the start of insulin until 1 hour after the fluids were initiated (timing generally relevant to management in ED).

Dose: $\mathbf{0 . 1} \mathbf{~ U} / \mathbf{k g} / \mathbf{h r}$ (order concentrated solution so that $1 \mathrm{U}=1 \mathrm{cc}$ )

For serum glucose less than $250-300 \mathrm{mg} / \mathrm{dl}$, add dextrose to IV fluids rather than lower the insulin infusion (see below under fluid management). The ultimate goal is for the serum glucoses to settle into the 100-200 range. When acidosis and/or wide anion gap has resolved, may decrease insulin infusion rate to $0.05 \mathrm{U} / \mathrm{kg} / \mathrm{hr}$. However, NEVER decrease insulin below $0.05 \mathrm{U} / \mathrm{kg} / \mathrm{hr}$. May also consider decreasing the insulin drip to $0.05 \mathrm{u} / \mathrm{kg} / \mathrm{hr}$ if glucose falling despite switching to all D10 NS, particularly if the last set of electrolytes suggest the DKA is resolving. Send electrolytes to confirm. (section "Completion of Therapy" for DKA resolution criteria) If resolution of the DKA stalls, consider increasing the insulin back to $0.1 \mathrm{u} / \mathrm{kg} / \mathrm{hr}$ and adjust glucose accordingly.

Do not stop IV insulin infusion until 15 - 30 minutes after subcutaneous insulin has been administered.

Fluid Management:

Initial fluid bolus of $10 \mathrm{cc} / \mathrm{kg}$ of normal saline or lactated Ringer (usually given in ED). Further fluid boluses reserved for patients in shock. Peripherally cool, mottled skin and dry oral muscosa from severe ketonemia may imitate shock state or severe dehydration and results in overhydration. A careful assessment of vital signs and examination of distal pulses should validate this assessment.

Sodium bicarbonate should never be used unless there is evidence of cardiac failure. Fluid composition 2 bag system:

Normal saline with 20 meq $\mathrm{KCl} \& 15 \mathrm{mmol} \mathrm{K}$ phos per liter (hold $\mathrm{K}$ for anuric patients or when serum $\mathrm{K}>5.5$ )

D10 normal saline with 20 meq $\mathrm{KCl} \& 15 \mathrm{mmol} \mathrm{K}$ phos per liter

Total fluid administration rate to equal $1.5 \mathrm{x}$ maintenance, where maintenance fluids are calculated using the weight-based formula: $100 \mathrm{cc} / \mathrm{kg} /$ day for the first $10 \mathrm{~kg}$ of bodyweight, 50 $\mathrm{cc} / \mathrm{kg} / \mathrm{day}$ for the second $10 \mathrm{~kg}$, and $20 \mathrm{cc} / \mathrm{kg} /$ day for weight $>20 \mathrm{kgs}$. In more severely dehydrated patients, fluids may be increased, but should never exceed a total of (initial boluses and maintenance) $4 \mathrm{~L} / \mathrm{m}^{2} /$ day. 
Initially, run the bag devoid of dextrose. As the blood glucose levels fall, but the need to treat the acidosis with an insulin drip persists, support the serum glucose by increasing the rate of the D10 solution while cutting back on the D0 solution. By varying the relative rates of the D0NS and D10NS solutions, any dextrose concentration between D0 and D10 may be administered. The goal of fluid therapy is to decrease the serum glucose by 50 to $100 \mathrm{mg} / \mathrm{dl} / \mathrm{hr}$ initially and then to maintain the glucose between $100-200 \mathrm{mg} / \mathrm{dl}$. Thus, obtain an accucheck glucose upon admission to the PICU. If accucheck has decreased by $>100 \mathrm{mg} / \mathrm{dl}$ from previous hour, increase glucose by $2.5 \%$ to a max of D10. For example, if the patient is on no dextrose, increase to D2.5; if on D5, go to D7.5; etc (see the table for sample calculations). Once the glucose has fallen below $300 \mathrm{mg} / \mathrm{dl}$, initiate or increase the dextrose infusion again to maintain the serum glucose between 100 to 200 $\mathrm{mg} / \mathrm{dl}$. Typical patients will initially start on the NS with K fluids, but then require increasing amounts of dextrose, with fluid infusion concentrations starting at D2.5 and progressing up through D5, D7.5 and possibly to D10. The total rate of fluid administration is always to remain at $1.5 \times$ maintenance - only the proportions from the NS and D10NS bags will change. Sample fluid rate calculations to make the various dextrose concentrations (D2.5, D5, D7.5) are shown in the following table and in the figure on the last page:

In a patient who needs $120 \mathrm{cc}$ per hour to make $1.5 \mathrm{x}$ maintenance:

\begin{tabular}{|l|l|l|}
\hline Final Glucose Conc. & Rate of NS w/K & Rate of D10 NS w/K \\
\hline D0 & 120 & 0 \\
\hline D2.5 & 90 & 30 \\
\hline D5 & 60 & 60 \\
\hline D7.5 & 30 & 90 \\
\hline D10 & 0 & 120 \\
\hline
\end{tabular}

If a higher concentration of glucose is needed, please be certain to order dextrose mixed in normal saline not in water. (D12.5 normal saline with 20 meq KCL \& 15mmol K phos/liter).

Monitoring:

Hourly neuro checks for all patients in DKA. Watch for signs of cerebral edema: decreasing sensorium, headaches, vomiting, pupillary changes, and seizures. Administer $1 \mathrm{~g} / \mathrm{kg}$ of mannitol, cut back fluid flow rates, manage ABCs, and contact ICU attending. Further points on cerebral edema are noted in Dr. Jones' handout.

Laboratory studies: hourly glucose, and q2h electrolytes for severe DKA patients $(\mathrm{pH}<7.1)$ and $\mathrm{q} 4 \mathrm{~h}$ for mild to moderate DKA. Space electrolytes to every 6 hours later as appropriate. In the presence of abnormal serum potassium $(\mathrm{K}>5.5$ or $<3.5)$, check $\mathrm{q} 2 \mathrm{~h} \mathrm{~K}$ with venous blood gas until normal. In the presence of hyperglycemia, the laboratory reported value for serum sodium is inaccurate and lower than the true serum sodium. The true serum sodium is calculated using the following factor: for each $100 \mathrm{mg} / \mathrm{dl}$ glucose above $100 \mathrm{mg} / \mathrm{dl}$, true serum sodium is 1.6 to $2.0 \mathrm{mEq} / \mathrm{L}$ above the laboratory reported sodium. As the serum glucose falls, the serum sodium should therefore rise along the same isobar as shown in Figure 2. A sodium 
that is not rising along the expected curve is concerning for SIADH and potential cerebral edema. In this instance, reconfirm the neuro exam and call the ICU attending for concerns.

\section{Completion of Therapy:}

Once nausea has resolved and mental status has improved, patients without any sign of cerebral edema may take clear liquids as diet colas in very limited amounts. Adjust IV infusion rates for $\mathrm{PO}$ intake.

Insulin infusion is complete at first mealtime (generally breakfast or dinner) after acidosis resolved (serum $\mathrm{HCO} 3>16$, venous $\mathrm{pH}>7.3$, and/or anion gap $\leq 15$ ).

Initiate home subcutaneous insulin regimen then serve meal and $\mathrm{d} / \mathrm{c}$ insulin drip. If patient is a new diabetic, see Addendum on insulin dosing in new diabetics.

Diet: Regular diet with 3 meals and 2-3 snacks at $1000 \mathrm{kcal}$ plus $100 \mathrm{kcal}$ for each year of age. ( 2 snacks for age $>10$ years; 3 for under). Please note, we no longer use the ADA diet Order blood sugar determinations before each meal, bedtime, and at $2 \mathrm{am}(7 \mathrm{am}, 11 \mathrm{am}, 5 \mathrm{pm}$, $9 \mathrm{pm}, 2 \mathrm{am})$.

Figure 1 (borrowed from: J Pediatr 1999;134:376-8): Schematic of two bag system and illustrative typical course. A, Two bag system allows independent manipulation of glucose and total fluid volume, because electrolyte content of 2 bags is identical except for dextrose. B, Differential rates of 2 bags modulates glucose delivery, which can be any concentration ranging from $0 \%$ to $10 \%$. Total fluid volume is based on patient's degree of dehydration and ongoing fluid requirement. $\mathbf{C}$, In this typical course, insulin therapy is instituted as continuous infusion of $0.1 \mathrm{U} / \mathrm{kg} / \mathrm{h}$, and total fluid rate is set at $200 \mathrm{cc} / \mathrm{h}$.

Because patient is markedly hyperglycemic, no dextrose is given initially. As insulin action lowers patient's blood glucose level, dextrose is titrated into IV fluid without changing administered fluid volume. Glucose titration aims to control rate of blood glucose decline (possible risk factor for cerebral edema) and prevent hypoglycemia in the face of continued insulin requirement. Later on, when patient's dehydration and ketosis become partially corrected, insulin and total fluid can also be independently adjusted 
Figure 1.

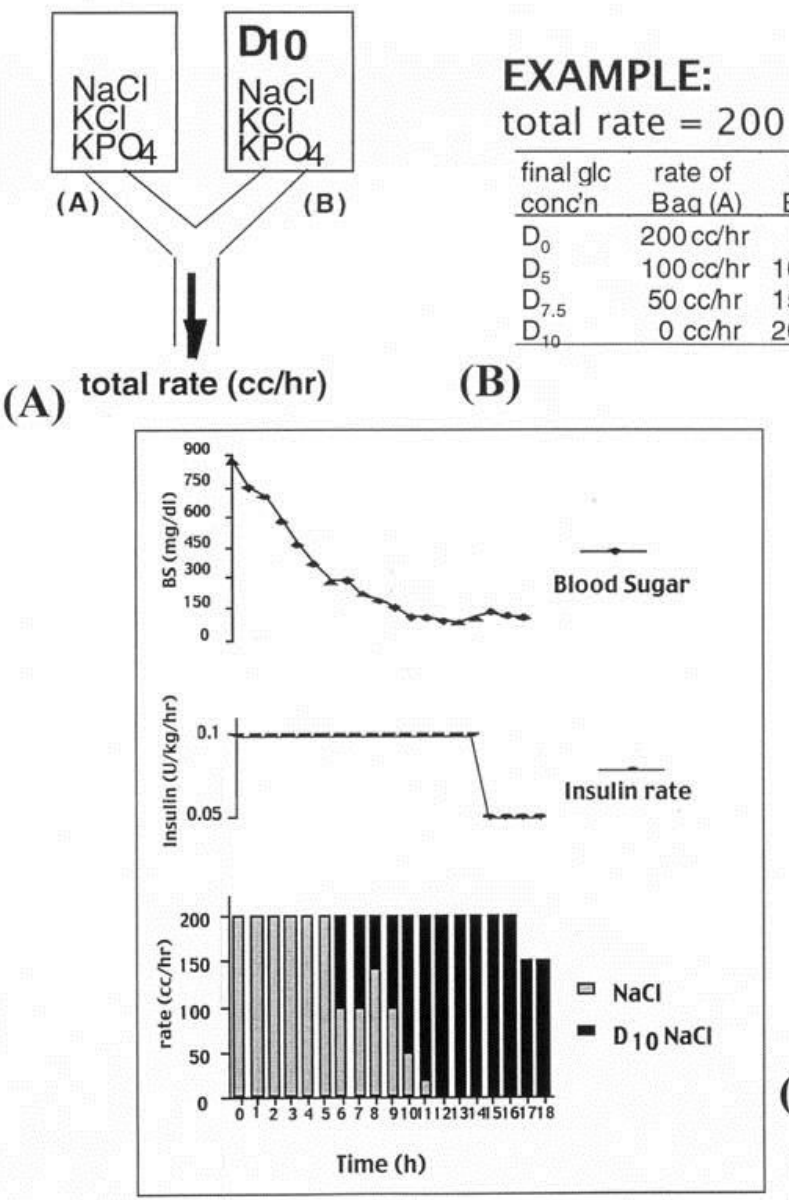

(C)

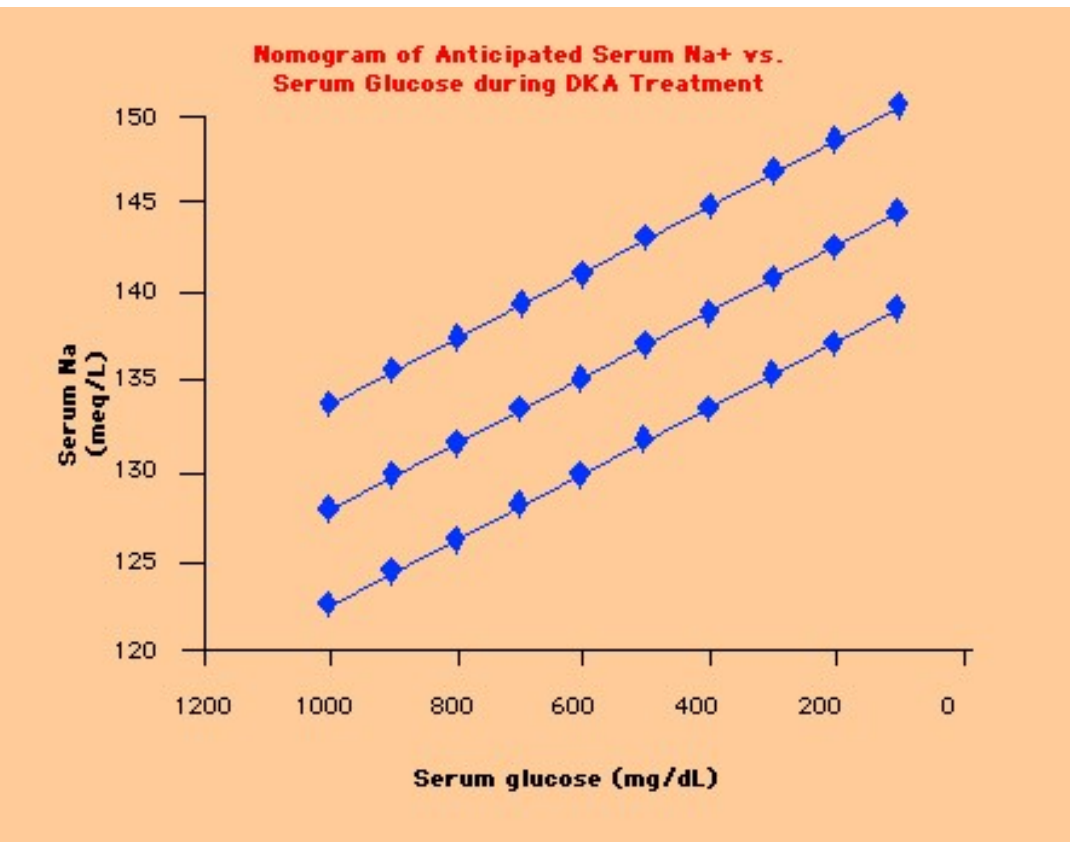

Figure 2 (from Uptodate.com) 
Appendix D3: Calculations for Fluid Maintenance and Replacement Volumes

\begin{tabular}{|c|c|c|c|c|c|c|c|}
\hline \multirow[t]{2}{*}{$\begin{array}{c}\text { Body } \\
\text { weight }(\mathrm{kg})\end{array}$} & \multirow[t]{2}{*}{$\mathrm{ml} / \mathrm{hr}$} & \multicolumn{2}{|c|}{$\begin{array}{c}\text { D 2.5\% } \\
\mathrm{ml} / \mathrm{hr}\end{array}$} & \multicolumn{2}{|c|}{$\begin{array}{l}\text { D 5\% } \\
\mathrm{ml} / \mathrm{hr}\end{array}$} & \multicolumn{2}{|c|}{$\begin{array}{c}\text { D 7.5\% } \\
\mathrm{ml} / \mathrm{hr}\end{array}$} \\
\hline & & NS & D $10 \%$ & NS & D $10 \%$ & NS & D $10 \%$ \\
\hline 4 & 26 & 19.5 & 6.5 & 13 & 13 & 6.5 & 19.5 \\
\hline 5 & 32 & 24 & 8 & 16 & 16 & 8 & 24 \\
\hline 6 & 38 & 28.5 & 9.5 & 19 & 19 & 9.5 & 28.5 \\
\hline 7 & 44 & 33 & 11 & 22 & 22 & 11 & 33 \\
\hline 8 & 50 & 37.5 & 12.5 & 25 & 25 & 12.5 & 37.5 \\
\hline 9 & 56 & 42 & 14 & 28 & 28 & 14 & 42 \\
\hline 10 & 64 & 48 & 16 & 32 & 32 & 16 & 48 \\
\hline 11 & 66 & 49.5 & 16.5 & 33 & 33 & 16.5 & 49.5 \\
\hline 12 & 70 & 52.5 & 17.5 & 35 & 35 & 17.5 & 52.5 \\
\hline 13 & 72 & 54 & 18 & 36 & 36 & 18 & 54 \\
\hline 14 & 76 & 57 & 19 & 38 & 38 & 19 & 57 \\
\hline 15 & 78 & 58.5 & 19.5 & 39 & 39 & 19.5 & 58.5 \\
\hline 16 & 82 & 61.5 & 20.5 & 41 & 41 & 20.5 & 61.5 \\
\hline 17 & 84 & 63 & 21 & 42 & 42 & 21 & 63 \\
\hline 18 & 88 & 66 & 22 & 44 & 44 & 22 & 66 \\
\hline 19 & 92 & 69 & 23 & 46 & 46 & 23 & 69 \\
\hline 20 & 94 & 70.5 & 23.5 & 47 & 47 & 23.5 & 70.5 \\
\hline 22 & 96 & 72 & 24 & 48 & 48 & 24 & 72 \\
\hline 24 & 100 & 75 & 25 & 50 & 50 & 25 & 75 \\
\hline 26 & 102 & 76.5 & 25.5 & 51 & 51 & 25.5 & 76.5 \\
\hline 28 & 104 & 78 & 26 & 52 & 52 & 26 & 78 \\
\hline 30 & 106 & 79.5 & 26.5 & 53 & 53 & 26.5 & 79.5 \\
\hline 32 & 110 & 82.5 & 27.5 & 55 & 55 & 27.5 & 82.5 \\
\hline 34 & 112 & 84 & 28 & 56 & 56 & 28 & 84 \\
\hline 36 & 114 & 85.5 & 28.5 & 57 & 57 & 28.5 & 85.5 \\
\hline 38 & 116 & 87 & 29 & 58 & 58 & 29 & 87 \\
\hline 40 & 120 & 90 & 30 & 60 & 60 & 30 & 90 \\
\hline 45 & 126 & 94.5 & 31.5 & 63 & 63 & 31.5 & 94.5 \\
\hline 50 & 132 & 99 & 33 & 66 & 66 & 33 & 99 \\
\hline 55 & 138 & 103.5 & 34.5 & 69 & 69 & 34.5 & 103.5 \\
\hline 60 & 144 & 108 & 36 & 72 & 72 & 36 & 108 \\
\hline 65 & 150 & 112.5 & 37.5 & 75 & 75 & 37.5 & 112.5 \\
\hline 70 & 156 & 117 & 39 & 78 & 78 & 39 & 117 \\
\hline 75 & 164 & 123 & 41 & 82 & 82 & 41 & 123 \\
\hline 80 & 170 & 127.5 & 42.5 & 85 & 85 & 42.5 & 127.5 \\
\hline
\end{tabular}




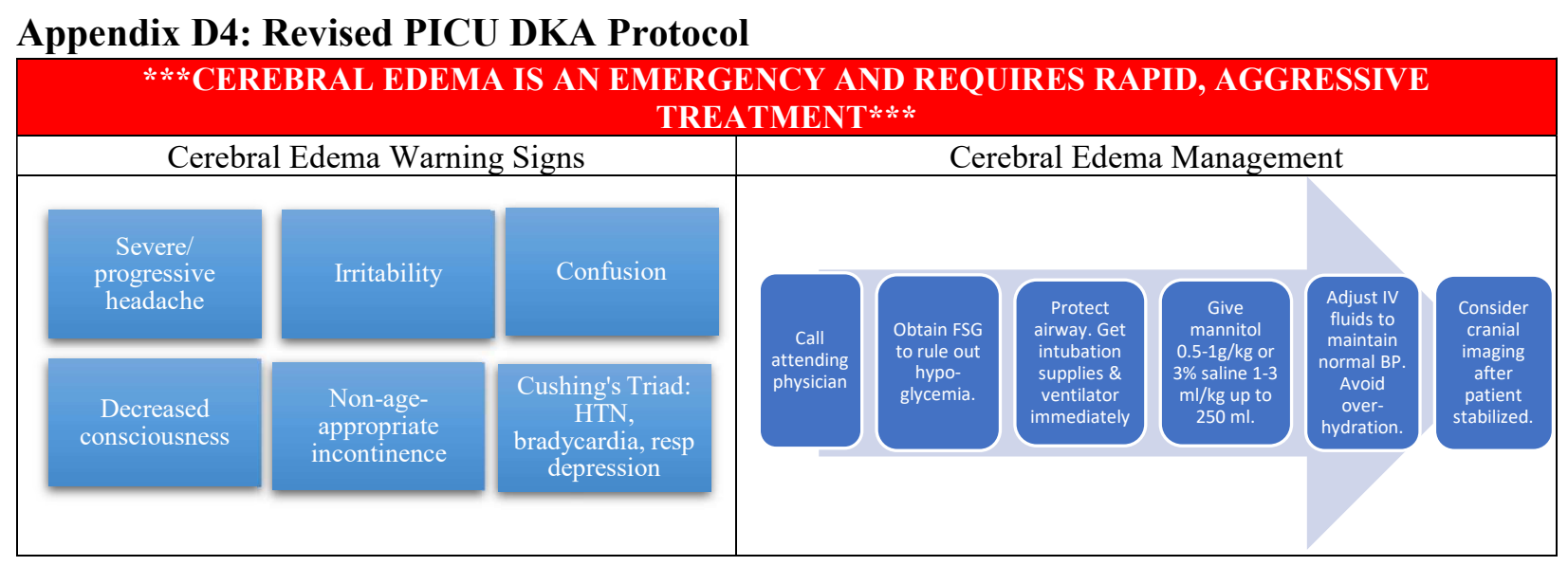

\begin{tabular}{|c|c|c|}
\hline \multicolumn{3}{|c|}{ RECOGNIZE DKA: Clinical history and signs } \\
\hline $\begin{array}{l}\text { Polyuria, Nocturia, and/or Enuresis } \\
\text { Polydipsia } \\
\text { Nausea and/or Vomiting } \\
\text { Abdominal pain }\end{array}$ & $\begin{array}{l}\text { Weakness } \\
\text { Fatigue/Lethargy/Drowsiness } \\
\text { Confusion } \\
\text { Decreased level of consciousness }\end{array}$ & $\begin{array}{l}\text { Dehydration } \\
\text { Deep sighing (Kussmaul) } \\
\text { Smell of ketones (acetone breath) } \\
\text { Sudden acute weight loss }\end{array}$ \\
\hline \multicolumn{3}{|c|}{ OBTAIN: Initial Labs } \\
\hline $\begin{array}{c}\text { Bedside glucose (BG) } \\
\text { Basic metabolic panel (BMP) }\end{array}$ & $\begin{array}{l}\text { Magnesium }(\mathrm{Mg}) \\
\text { Phosphate }\left(\mathrm{PO}_{4}\right)\end{array}$ & $\begin{array}{c}\text { Hemoglobin A1c } \\
\text { Beta-hydroxybutyrate (BHB) } \\
\text { Venous blood gas (VBG) }\end{array}$ \\
\hline \multicolumn{3}{|c|}{ Diagnostic lab criteria } \\
\hline $\begin{array}{c}\text { Hyperglycemia } \\
\mathrm{BHB} \geq 3 \mathrm{mmol} / \mathrm{L}\end{array}$ & $\mathrm{pH}<7.3$ & Bicarbonate $(\mathrm{HCO} 3)<15$ \\
\hline
\end{tabular}

Observe PALS emergency assessment and resuscitation guidelines as needed.

Fluid resuscitation: Give $10 \mathrm{ml} / \mathrm{kg}$ bolus of NS or LR (usually given in ED) over 1 hour. May repeat once as needed (do not exceed $20 \mathrm{ml} / \mathrm{kg}$ total unless patient is in shock and without approval from attending physician). Monitor ECG for abnormal T-waves. Do not give a bicarbonate bolus unless specified by attending, which would be a rare circumstance.

\begin{tabular}{|c|c|c|}
\hline Insulin & Maintenance Fluid Therapy & Monitor: \\
\hline $\begin{array}{l}\text { Continuous insulin infusion (dilute } \\
\text { insulin to } 1 \text { unit } / \mathrm{ml} \text { ). Run insulin at } \\
0.1 \text { units } / \mathrm{kg} / \text { hour }\end{array}$ & $\begin{array}{l}\text { Start MIVF at } 1.5 \text { maintenance (see } \\
\text { Calculations for Fluid Maintenance and } \\
\text { Replacement Volumes table). Start with } \\
\text { isotonic crystalloid with no dextrose } \\
\text { (NS+20KCl+15Kphos). Have a bag of same } \\
\text { isotonic crystalloid with } 10 \% \text { dextrose added } \\
\text { (D10NS+20KCl+15Kphos) connected in line } \\
\text { but not running. *Hold K for anuric patients } \\
\text { or if serum K >5.5. } \\
\text { *NOTE: In referring ED's it is appropriate } \\
\text { to start patient on NS and progress to D5NS } \\
\text { if the glucose is trending down. Fluids will } \\
\text { be changed to include K in PICU. }\end{array}$ & $\begin{array}{l}\text { Hourly BG; hourly input \& output; } \\
\text { hourly neuro status; continuous ECG } \\
\text { for T-wave changes; electrolytes with } \\
\text { anion gap, } \mathrm{Mg}, \mathrm{PO}_{4} \text { every } 2 \text { hours if } \\
\text { initial VBG revealed } \mathrm{pH}<7.1 \text { or } \\
\text { every } 4 \text { hours if } \mathrm{pH}>7.1 \\
\text { (See above for cerebral edema } \\
\text { warning signs and treatment) }\end{array}$ \\
\hline
\end{tabular}

When BG drops by more than $\mathbf{9 0} \mathbf{~ m g / d L}$ in 1 hour or falls below $\mathbf{3 0 0} \mathbf{~ m g / d L}$, start D 10\% solution and decrease NS solution according to Calculations for Fluid Maintenance and Replacement Volumes table (Start at D2.5\% and reassess in 1 hour). As BG falls, continue to add dextrose according to Calculations for Fluid Maintenance and Replacement Volumes table (D5\%, D7.5\%, D10\%). Do not stop insulin drip. (See next page for algorithm) 


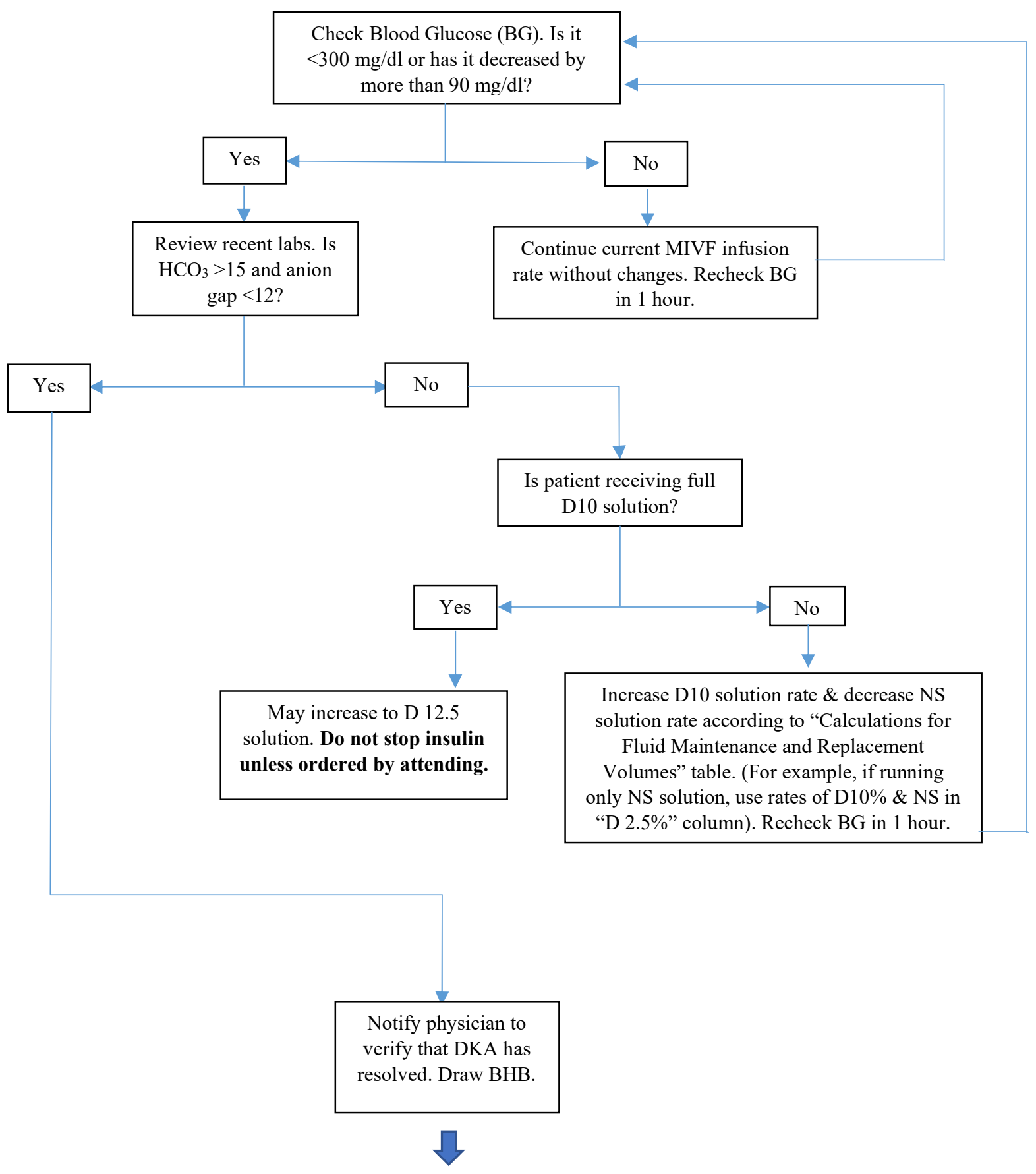

Completion of Therapy: Once HCO3 $>15$ and anion gap $<12, \mathrm{pH}>7.3$, and BG is $150-200 \mathrm{mg} / \mathrm{dL}$, plan to stop fluids. If transitioning off of insulin at night, consider running Plasmalyte without dextrose overnight. Obtain a BHB once more (as shown in algorithm) to verify ketoacidosis is resolved (ideally with 4 am labs if planning to serve patient breakfast). Administer longacting subcutaneous (SC) insulin (i.e. Lantus, Levemir, Basaglar) if not already administered within the last 24 hours and fastacting insulin (i.e. Humalog or Novolog) at least 15 minutes before stopping continuous IV insulin. If patient uses insulin pump, use new tubing and connection site. *Make every effort to administer long-acting insulin as close to the time of regular dose as possible (i.e. if a patient usually receives a dose at night, administer at night, even if patient is still on IV insulin drip). 


\begin{tabular}{|c|c|c|c|c|c|c|c|c|}
\hline \multirow{3}{*}{$\begin{array}{c}\text { Body weight } \\
(\mathrm{kg})\end{array}$} & \multicolumn{8}{|c|}{ Calculations for Fluid Maintenance and Replacement Volumes } \\
\hline & \multirow[t]{2}{*}{$\begin{array}{l}\mathrm{MIVF} \\
\mathrm{ml} / \mathrm{hr}\end{array}$} & \multirow[t]{2}{*}{$\begin{array}{c}1.5 \mathrm{X} \mathrm{MIVF} \\
\mathrm{ml} / \mathrm{hr}\end{array}$} & \multicolumn{2}{|c|}{$\begin{array}{c}\mathrm{D} 2.5 \% \\
\mathrm{ml} / \mathrm{hr}\end{array}$} & \multicolumn{2}{|c|}{$\begin{array}{l}\mathrm{D} 5 \% \\
\mathrm{ml} / \mathrm{hr}\end{array}$} & \multicolumn{2}{|c|}{$\begin{array}{c}\mathrm{D} 7.5 \% \\
\mathrm{ml} / \mathrm{hr}\end{array}$} \\
\hline & & & NS & D $10 \%$ & NS & D $10 \%$ & NS & D $10 \%$ \\
\hline 4 & 17 & 25 & 19 & 6 & 12.5 & 12.5 & 6 & 19 \\
\hline 5 & 21 & 32 & 24 & 8 & 16 & 16 & 8 & 24 \\
\hline 6 & 25 & 38 & 28.5 & 9.5 & 19 & 19 & 9.5 & 28.5 \\
\hline 7 & 29 & 44 & 33 & 11 & 22 & 22 & 11 & 33 \\
\hline 8 & 33 & 50 & 37.5 & 12.5 & 25 & 25 & 12.5 & 37.5 \\
\hline 9 & 37 & 56 & 42 & 14 & 28 & 28 & 14 & 42 \\
\hline 10 & 42 & 63 & 47.5 & 15.5 & 31.5 & 31.5 & 15.5 & 47.5 \\
\hline 11 & 44 & 66 & 49.5 & 16.5 & 33 & 33 & 16.5 & 49.5 \\
\hline 12 & 46 & 69 & 52 & 17 & 34.5 & 34.5 & 17 & 52 \\
\hline 13 & 48 & 72 & 54 & 18 & 36 & 36 & 18 & 54 \\
\hline 14 & 50 & 75 & 56 & 19 & 38 & 38 & 19 & 56 \\
\hline 15 & 52 & 78 & 58.5 & 19.5 & 39 & 39 & 19.5 & 58.5 \\
\hline 16 & 54 & 81 & 61 & 20 & 41 & 41 & 20 & 61 \\
\hline 17 & 56 & 84 & 63 & 21 & 42 & 42 & 21 & 63 \\
\hline 18 & 58 & 87 & 65 & 22 & 44 & 44 & 22 & 65 \\
\hline 19 & 60 & 90 & 67.5 & 22.5 & 46 & 46 & 22.5 & 67.5 \\
\hline 20 & 63 & 94 & 70.5 & 23.5 & 47 & 47 & 23.5 & 70.5 \\
\hline 22 & 64 & 96 & 72 & 24 & 48 & 48 & 24 & 72 \\
\hline 24 & 66 & 99 & 74 & 25 & 50 & 50 & 25 & 74 \\
\hline 26 & 68 & 102 & 76.5 & 25.5 & 51 & 51 & 25.5 & 76.5 \\
\hline 28 & 69 & 104 & 78 & 26 & 52 & 52 & 26 & 78 \\
\hline 30 & 71 & 107 & 80 & 27 & 53 & 53 & 27 & 80 \\
\hline 32 & 73 & 110 & 82.5 & 27.5 & 55 & 55 & 27.5 & 82.5 \\
\hline 34 & 74 & 111 & 83 & 28 & 56 & 56 & 28 & 83 \\
\hline 36 & 76 & 114 & 85.5 & 28.5 & 57 & 57 & 28.5 & 85.5 \\
\hline 38 & 78 & 117 & 88 & 29 & 58 & 58 & 29 & 88 \\
\hline 40 & 79 & 119 & 89 & 30 & 60 & 60 & 30 & 89 \\
\hline 45 & 83 & 125 & 94 & 31 & 63 & 63 & 31 & 94 \\
\hline 50 & 88 & 132 & 99 & 33 & 66 & 66 & 33 & 99 \\
\hline 55 & 92 & 138 & 103.5 & 34.5 & 69 & 69 & 34.5 & 103.5 \\
\hline 60 & 96 & 144 & 108 & 36 & 72 & 72 & 36 & 108 \\
\hline 65 & 100 & 150 & 112.5 & 37.5 & 75 & 75 & 37.5 & 112.5 \\
\hline 70 & 104 & 156 & 117 & 39 & 78 & 78 & 39 & 117 \\
\hline 75 & 108 & 162 & 121.5 & 40.5 & 82 & 82 & 41 & 123 \\
\hline 80 & 113 & 170 & 127.5 & 42.5 & 85 & 85 & 42.5 & 127.5 \\
\hline 85 & 117 & 176 & 132 & 44 & 88 & 88 & 44 & 132 \\
\hline 90 & 121 & 182 & 136.5 & 45.5 & 91 & 91 & 45.5 & 136.5 \\
\hline 95 & 125 & 188 & 141 & 47 & 94 & 94 & 47 & 141 \\
\hline 100 & 129 & 194 & 145.5 & 48.5 & 97 & 97 & 48.5 & 145.5 \\
\hline
\end{tabular}




\section{Tips for Physicians:}

1. Place orders for insulin and fluids STAT so Pharmacy sees them sooner. Call Pharmacy as well.

2. When patient is newly diagnosed with diabetes, be sure to get these 1-time labs: GAD antibodies, TSH, free T4, celiac panel, and islet cell autoantibodies. Whether patient has new onset or is known to have diabetes, always get A1c.

3. In hyperglycemia, the lab-reported serum sodium is inaccurate \& lower than the true serum sodium. For every $100 \mathrm{mg} / \mathrm{dL}$ glucose above $100 \mathrm{mg} / \mathrm{dL}$, true serum sodium is 1.6 to $2.0 \mathrm{mEq} / \mathrm{L}$ above reported value. (For example, if patient's blood glucose is 300 $\mathrm{mg} / \mathrm{dL}$, add 3.2 to $4 \mathrm{mEq} / \mathrm{L}$ to the lab-reported value). If serum sodium values do not rise as hyperglycemia is corrected, this may be an early sign that patient is at risk for cerebral injury.

4. Currently, the PICU uses NS $+20 \mathrm{mEq}$ of potassium chloride $(\mathrm{KCl})+15 \mathrm{mmol}$ of potassium phosphate (K Phos) and $10 \%$ dextrose in $\mathrm{NS}+20 \mathrm{mEq} \mathrm{KCl}+15 \mathrm{mmol} \mathrm{K}$ Phos. This compensates for potassium \& phosphate lost in blood from polyuria, emesis, and reuptake by cells in the presence of insulin. Do not add $\mathrm{KCl} \& \mathrm{~K}$ Phos to fluids if serum potassium is $>5.5$.

5. Stopping the insulin drip is the last course of action if glucose levels are falling too quickly or patient's glucose falls below $150 \mathrm{mg} / \mathrm{dL}$. Insulin is treating both the hyperglycemia and the ketoacidosis. First, max out dextrose concentration in MIVF. For a peripheral IV, it is safe to go as high as $12.5 \%$ dextrose. Place STAT order for new bag of $12.5 \%$ Dextrose in NS $+20 \mathrm{mEq} \mathrm{KCl}+15 \mathrm{mmol} \mathrm{K}$ Phos. If this is not enough to stabilize blood glucose levels, reduce insulin to $0.05 \mathrm{units} / \mathrm{kg} / \mathrm{hr}$. If blood glucose is still precipitously dropping (dropping by more than $90 \mathrm{mg} / \mathrm{dL} / \mathrm{hour}$ ) or falls below $100 \mathrm{mg} / \mathrm{dL}$ at maxed dextrose concentration, notify attending.

6. As the patient receives insulin, hypokalemia is likely to occur. That is why the MIVF includes $\mathrm{KCl}$ and $\mathrm{K}$ Phos. However, if the patient has a $\mathrm{K}>5.5$, exclude $\mathrm{K}$ from MIVF until needed per attending's order.

7. If the patient has a new onset of diabetes or has established diabetes and uses long-acting insulin, administer a dose of long-acting insulin at night (usual time patient would receive long-acting insulin if not on protocol), even if patient is still on continuous insulin drip. Studies have shown it is safe for patient to receive long-acting insulin more than 4 hours before stopping the continuous insulin drip.

8. There are certain patients that require more close attention because these are some of the cases that have the highest risk for complications and poor outcomes. These include patients with a $\mathrm{pH}<6.9$, major weight loss at onset of new diagnosis, children who have received excessive amounts of IVF or hypotonic fluids prior to transfer, and children with high glucose with a normal or high sodium. 


\section{Tips for New Nurses (or Experienced Nurses who would like a review):}

1. Equipment that should be in your patient's room on admission: 3 maintenance fluid pumps with cords (a 3-pump connector cord if available and a wall cord), IV tubing for 3 bags of fluid, octopus with 3 ports (or 2 Y connectors if you don't have a 3-port connector), bottle of glucometer strips, glucometer, cup of lancets, cup of green top microtainers, blood gas tubes, syringes and flushes, IV set up, hat or urinal for careful I\&O monitoring, telemetry leads, alcohol swabs. Make sure the fluids and insulin drip have been ordered STAT. If you receive them before the patient arrives, prime your tubing, connect to octopus, and set up pumps. Use Calculations for Fluid Maintenance and Replacement Volumes table to determine MIVF rate. Most likely, you will start the NS bag at the value in the $\mathrm{ml} / \mathrm{hr}$ column based on your patient's weight unless their BG on arrival $<300 \mathrm{mg} / \mathrm{dL}$ and/or $\mathrm{HCO}_{3}>15$.

2. If the patient is stable upon arrival, offer restroom before settling patient in and putting on telemetry leads and changing fluid bags and tubing.

3. If possible, make sure your patient has at least 2 IV's, preferably at least one that draws. Try to have them in different limbs or at least different veins. That way you can run fluids through one IV and draw labs through the other without having to stop fluids or stick your patient. You will be drawing a lot of labs until their ketoacidosis is corrected. Draw a large waste if you have to draw labs from the line where fluids are infusing.

4. If you suspect cerebral edema, notify the provider ASAP. It is better to be over-cautious and wrong than under-cautious and wrong.

5. If you have not done so already, read through the Tips for Physicians page. It will give you a better understanding of why the protocol is as it is and why the physicians are ordering what they order.

6. If there are concepts about DKA and its management that you don't understand, ask questions and do research. Knowledge will help you to be a stronger advocate for your patient.

References:

Harrison, V. S., Rustico, S., Palladino, A. A., Ferrara, C., \& Hawkes, C. P. (2017). Glargine co

administration with intravenous insulin in pediatric diabetic ketoacidosis is safe and facilitates transition to a subcutaneous regimen. Pediatric Diabetes, 18(8), 742-748. Retrieved June 29, 2019 from https://doiorg.www.libproxy.wvu.edu/10.1111/pedi.12462

Shankar, V., Haque, A., Churchwell, K. B., \& Russell, W. (2007). Insulin glargine supplementation during early management phase of diabetic ketoacidosis in children. Intensive Care Medicine, 33(7), 1173-1178. https://doi-org.www.libproxy.wvu.edu/10.1007/s00134-007-0674-3

Staff of WVUM PICU. (n.d.). WVU PICU DKA protocol.

Wolfsdorf, J. I., Glaser, N., Agus, M., Fritsch, M., Hanas, R., Rewers, A., ... Codner, E. (2018). ISPAD Clinical Practice Consensus Guidelines 2018: Diabetic ketoacidosis and the hyperglycemic hyperosmolar state. Pediatric Diabetes, 19, 155-177. Retrieved June 29, 2019 from https://doiorg.www.libproxy.wvu.edu/10.1111/pedi.12701 


\section{Appendix E: Staff Survey}

Please select one answer that best describes your current position:

Physician

Physician's Assistant

Nurse Practitioner

Nurse

Pharmacist

Medical Student/Resident

Other (please specify):

How long have you worked in the PICU?

$0-2$ years

2-5 years

5-10 years

10 or more years

Have you ever used the previous DKA protocol?
Yes
No

How familiar are you with the revisions to the DKA protocol? (Beginning of Implementation Survey only)

$\square$ This is the first I am hearing about the DKA protocol revisions.

I have heard that the DKA protocol was being revised, but I don't know anything about the changes yet.

I have seen the PowerPoint educational presentation about the revisions being made to the DKA protocol in person.

I have seen a recording of the PowerPoint educational presentation about the revisions being made to the DKA protocol.

How familiar are you with the revisions to the DKA protocol? (End of Implementation Survey only)

$\square$ This is the first I am hearing about the DKA protocol revisions.

$\square$ I have heard that the DKA protocol was being revised, but I don't know anything about the changes yet.

$\square$ I have seen the PowerPoint educational presentation about the revisions being made to the DKA protocol but have not used the revised protocol yet.

I have seen the PowerPoint educational presentation about the revisions being made to the DKA protocol and have worked with the revised protocol.

I did not see the PowerPoint educational presentation about the revisions being made to the DKA protocol but have worked with the revised protocol.

How well do you think each is followed by staff? 


\begin{tabular}{|l|l|l|l|l|l|l|l|}
\hline & $\begin{array}{c}\text { Extremely } \\
\text { Well }\end{array}$ & $\begin{array}{c}\text { Very } \\
\text { Well }\end{array}$ & $\begin{array}{c}\text { Moderately } \\
\text { Well }\end{array}$ & $\begin{array}{c}\text { Slightly } \\
\text { Well }\end{array}$ & $\begin{array}{c}\text { Not Well } \\
\text { at All }\end{array}$ & $\begin{array}{c}\text { Have Not } \\
\text { Had a } \\
\text { Chance to } \\
\text { Use Yet }\end{array}$ & Unsure \\
\hline $\begin{array}{l}\text { Previous } \\
\text { DKA } \\
\text { Protocol }\end{array}$ & & & & & & & \\
\hline $\begin{array}{l}\text { New DKA } \\
\text { Protocol }\end{array}$ & & & & & & & \\
\hline
\end{tabular}

Rate ease of use for each of the following:

\begin{tabular}{|l|l|l|l|l|l|l|}
\hline & Very easy & easy & $\begin{array}{c}\text { Neither easy } \\
\text { nor } \\
\text { complicated }\end{array}$ & Complicated & $\begin{array}{c}\text { Very } \\
\text { complicated }\end{array}$ & $\begin{array}{c}\text { Did Not } \\
\text { Use }\end{array}$ \\
\hline $\begin{array}{l}\text { Previous } \\
\text { DKA } \\
\text { protocol }\end{array}$ & & & & & & \\
\hline $\begin{array}{l}\text { New DKA } \\
\text { protocol }\end{array}$ & & & & & & \\
\hline
\end{tabular}

\begin{tabular}{|l|c|c|c|c|c|}
\hline & $\begin{array}{c}\text { Extremely } \\
\text { Likely }\end{array}$ & $\begin{array}{c}\text { Somewhat } \\
\text { Likely }\end{array}$ & $\begin{array}{c}\text { Neither Likely } \\
\text { nor Unlikely }\end{array}$ & $\begin{array}{c}\text { Somewhat } \\
\text { unlikely }\end{array}$ & $\begin{array}{c}\text { Extremely } \\
\text { Unlikely }\end{array}$ \\
\hline $\begin{array}{l}\text { How likely are } \\
\text { you to use the } \\
\text { new protocol? }\end{array}$ & & & & & \\
\hline
\end{tabular}

\begin{tabular}{|l|l|l|l|l|l|}
\hline & $\begin{array}{c}\text { Extremely } \\
\text { Well }\end{array}$ & Very Well & Moderately Well & Slightly Well & Not Well at all \\
\hline $\begin{array}{l}\text { How well does } \\
\text { the new } \\
\text { protocol follow } \\
\text { your expected } \\
\text { treatment plan } \\
\text { for DKA? }\end{array}$ & & & & & \\
& & & & & \\
\hline
\end{tabular}

What is something you like about the previous protocol? 
What is something you would change about the previous protocol?

What is something you like about the new protocol?

What is something you would change about the new protocol?

From what you've seen, do you prefer the old DKA protocol or the new DKA protocol? Why? 


\section{Appendix F1: Project Timeline}

in

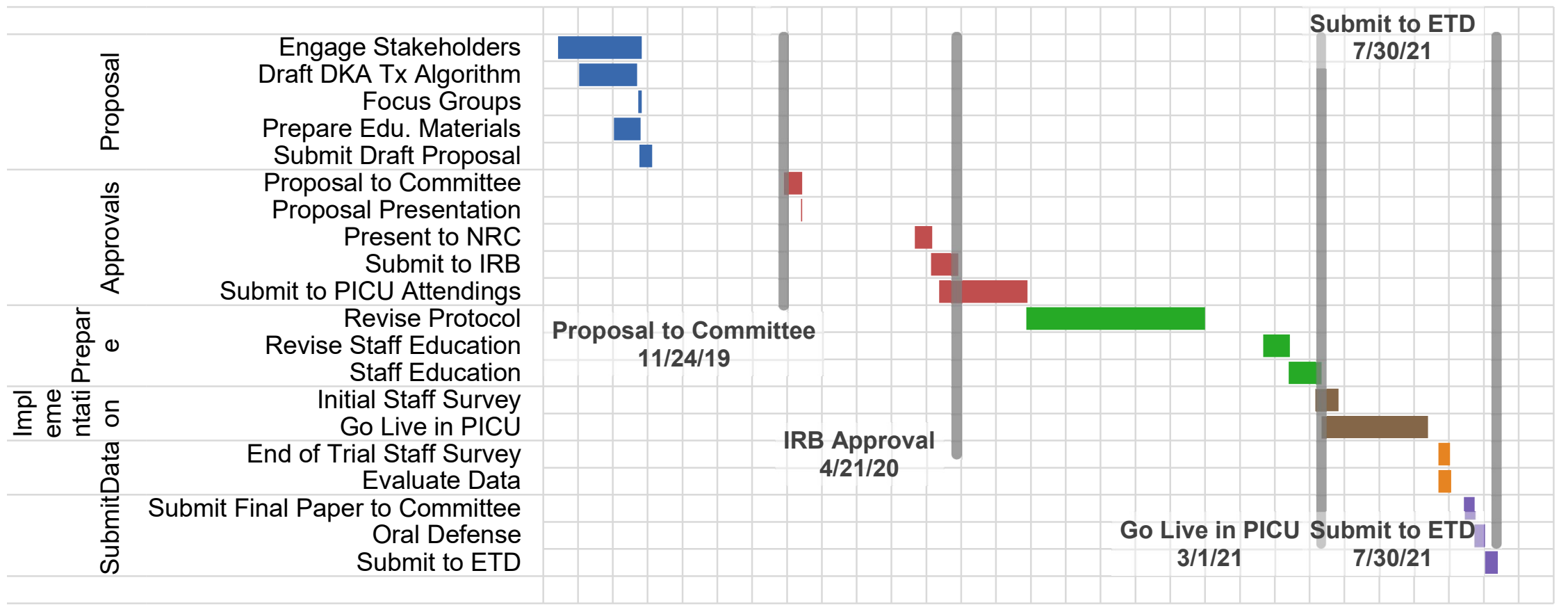


Appendix F2: Project Timeline Table

\begin{tabular}{|c|c|c|c|}
\hline Proposal & Engage Stakeholders & $5 / 14 / 19$ & $7 / 24 / 19$ \\
\hline & Draft DKA Treatment Algorithm & 6/1/19 & $7 / 20 / 19$ \\
\hline & Focus Groups & $7 / 22 / 19$ & $7 / 24 / 19$ \\
\hline & Prepare Edu. Materials & $7 / 1 / 19$ & $7 / 23 / 19$ \\
\hline & Submit Draft Proposal & $7 / 23 / 19$ & $8 / 2 / 19$ \\
\hline \multirow[t]{5}{*}{ Approvals } & Proposal to Committee & $11 / 24 / 19$ & $12 / 9 / 19$ \\
\hline & Proposal Presentation & $12 / 9 / 19$ & $12 / 9 / 19$ \\
\hline & Present to NRC & $3 / 16 / 20$ & $3 / 30 / 20$ \\
\hline & Submit to IRB & $3 / 30 / 20$ & $4 / 21 / 20$ \\
\hline & Submit to PICU Attending Physician & $4 / 6 / 20$ & $6 / 20 / 20$ \\
\hline \multirow[t]{3}{*}{ Prepare } & Revise Protocol & $6 / 20 / 20$ & $11 / 20 / 20$ \\
\hline & Revise Staff Education & $1 / 10 / 21$ & $2 / 1 / 21$ \\
\hline & Staff Education & $2 / 1 / 21$ & $2 / 28 / 21$ \\
\hline \multirow[t]{2}{*}{ Implementation } & Initial Staff Survey & $2 / 24 / 21$ & $3 / 15 / 21$ \\
\hline & Go Live in PICU & $3 / 1 / 21$ & $5 / 31 / 21$ \\
\hline \multirow[t]{2}{*}{ Data } & End of Trial Staff Survey & $6 / 10 / 21$ & $6 / 19 / 21$ \\
\hline & Evaluate Data & $6 / 10 / 21$ & $6 / 20 / 21$ \\
\hline \multirow[t]{3}{*}{ Submit } & Submit Final Paper to Committee & $7 / 2 / 21$ & $7 / 10 / 21$ \\
\hline & Oral Defense & $7 / \mathbf{1 0} / 21$ & $7 / 19 / 21$ \\
\hline & Submit to ETD & $7 / 20 / 21$ & $7 / 30 / 21$ \\
\hline
\end{tabular}




\section{Appendix G: Survey Results Graphs}

Who took the initial survey?

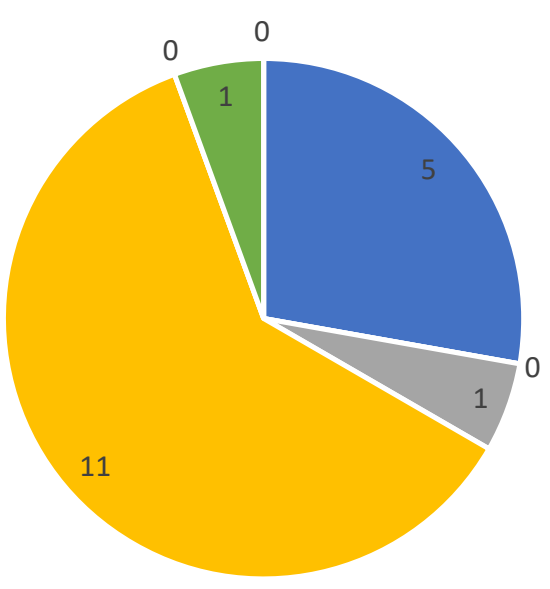

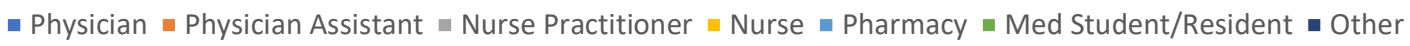

\section{Who took the final survey?}

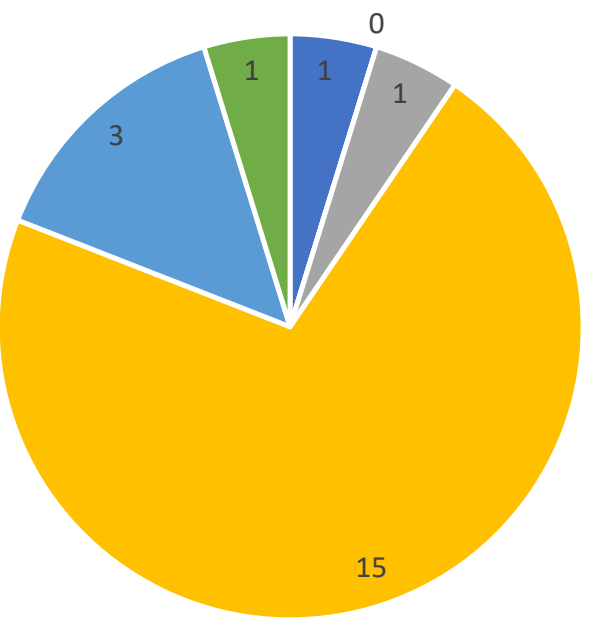

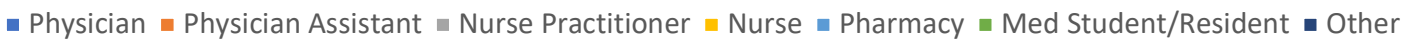




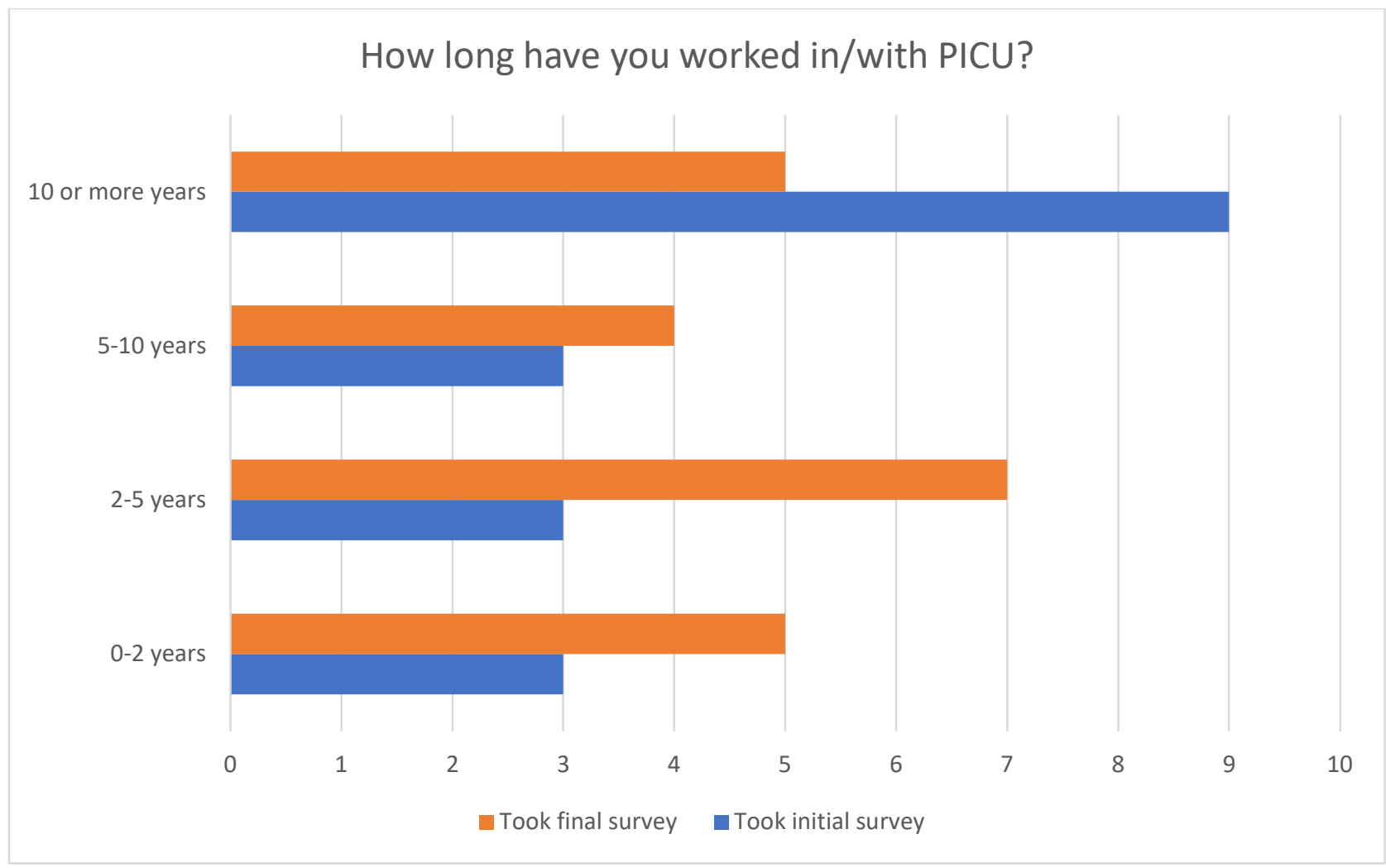

\section{How Well Does New Protocol Follow Expected Treatment Plan for Managing DKA?}

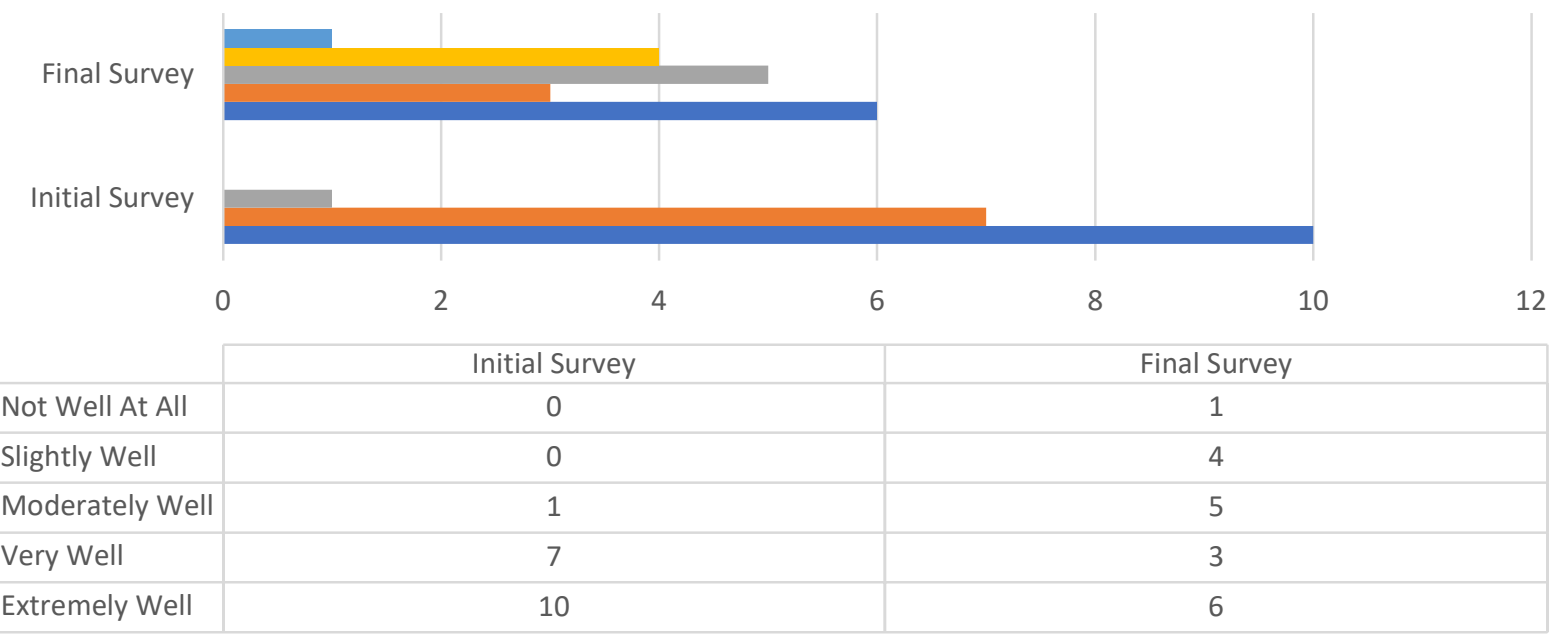

Not Well At All Slightly Well $\square$ Moderately Well $\square$ Very Well $\square$ Extremely Well 


\section{How Well is Each Protocol Followed by Staff?}

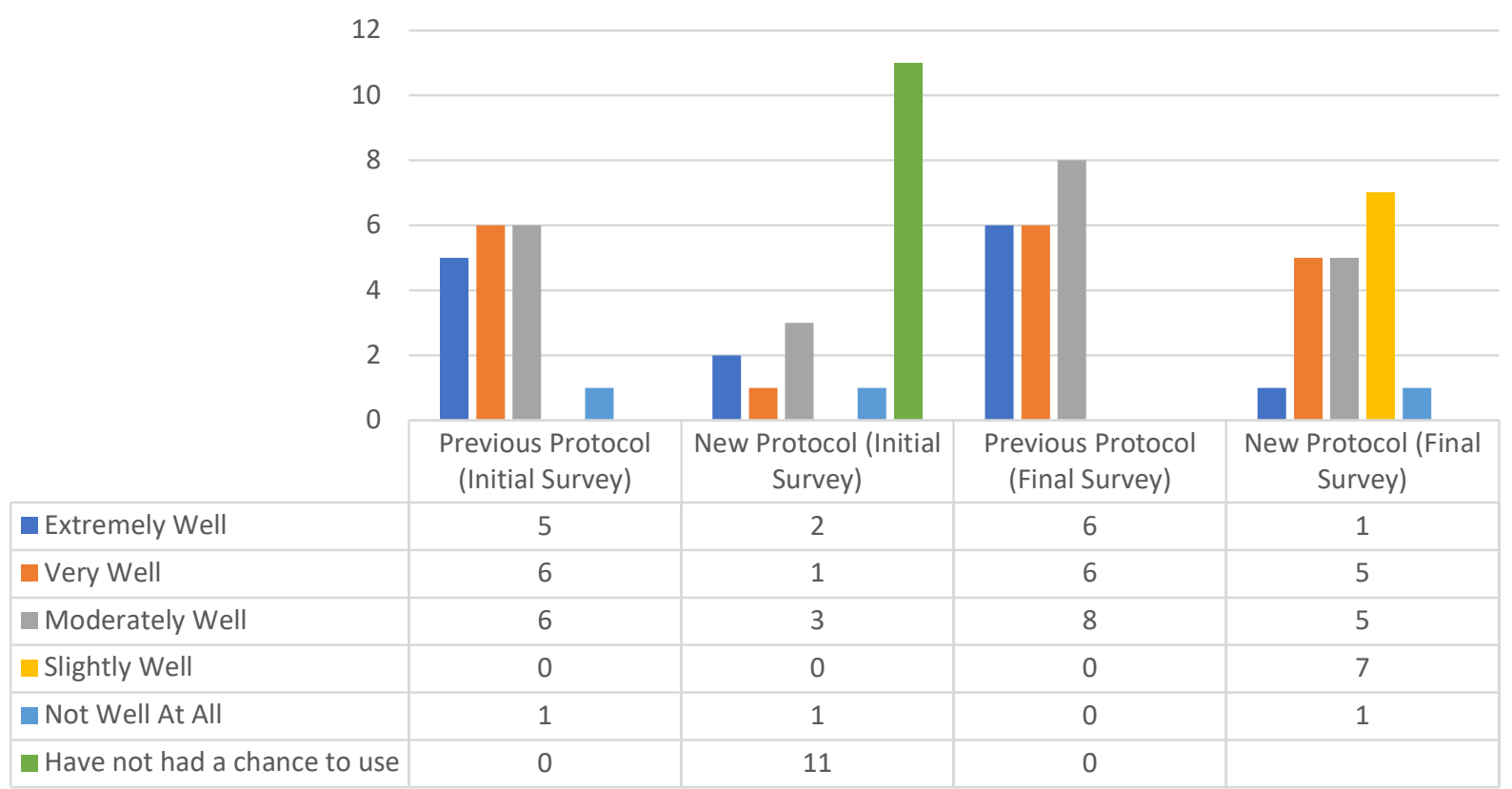

Extremely Well $\square$ Very Well $\square$ Moderately Well $\square$ Slightly Well $\square$ Not Well At All $\square$ Have not had a chance to use

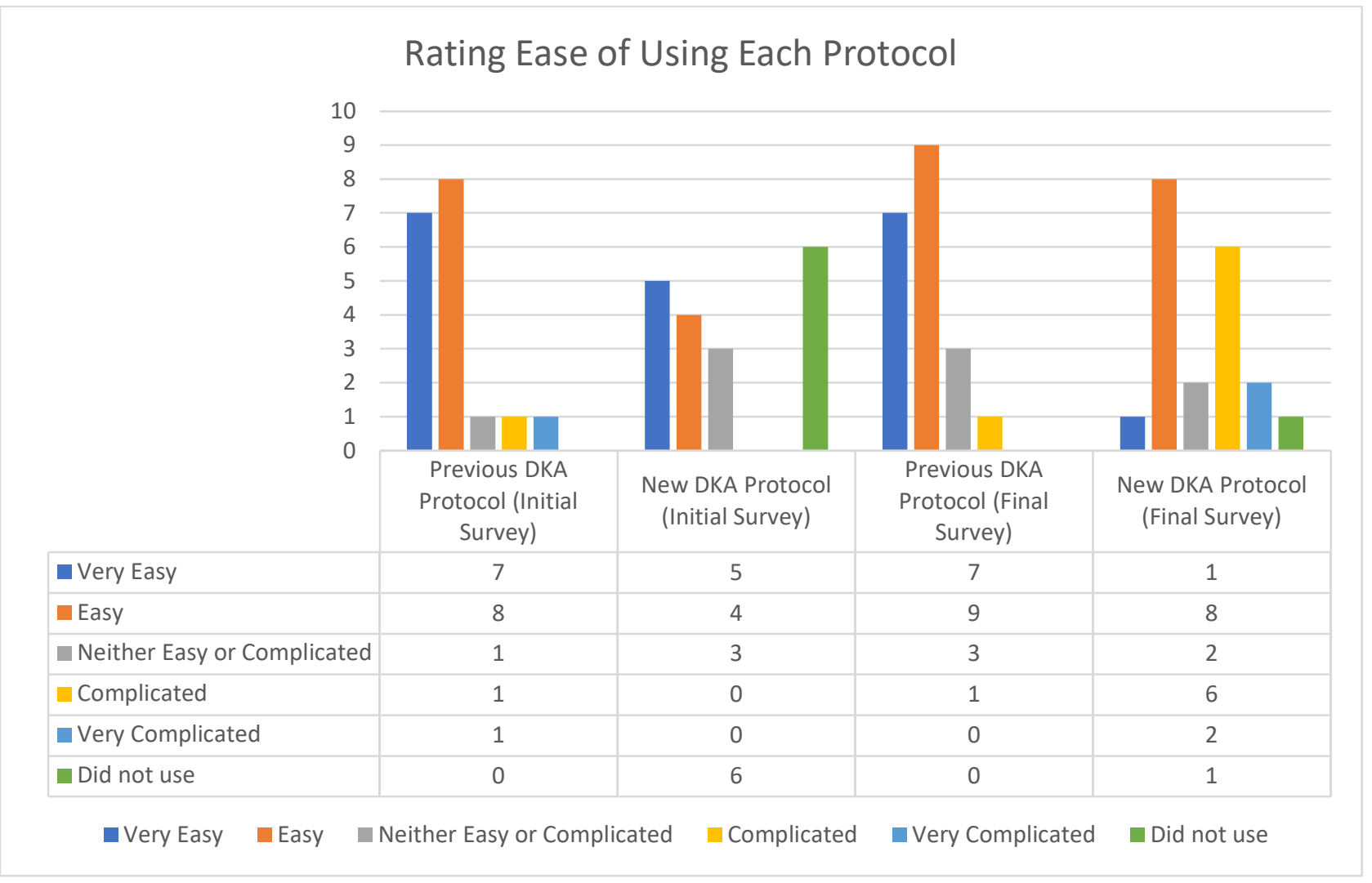




\section{How likely are you to use the new protocol?}

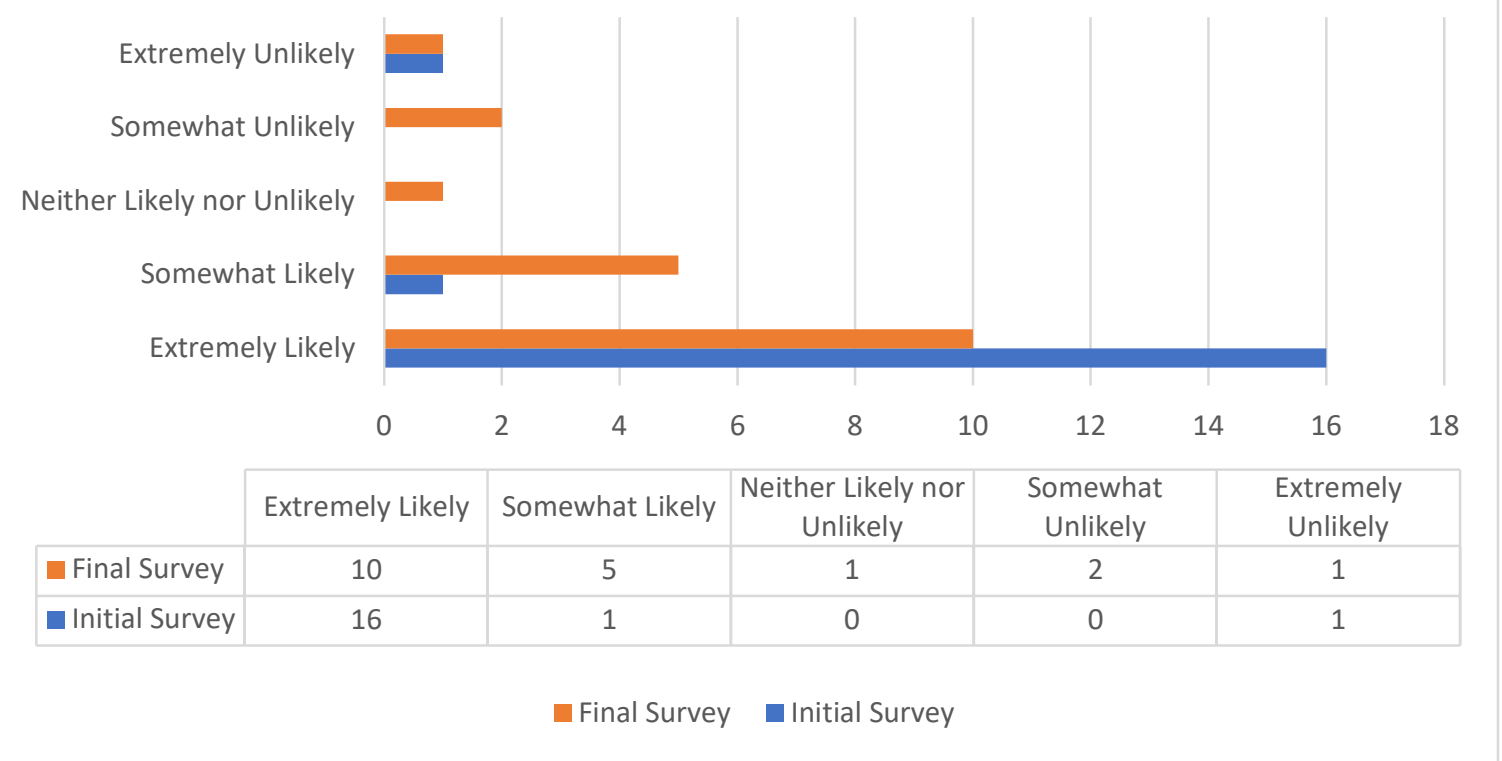




\section{Appendix H: Themes of Open-Ended Questions of Surveys}

Question: What is something you like about the previous protocol?

\begin{tabular}{|l|l|}
\hline Initial survey & Final survey \\
\hline $\begin{array}{l}\text { Nothing: "Nothing-extremely confusing for } \\
\text { both nursing staff and residents. Even when } \\
\text { nursing is fluent in use, making residents and } \\
\text { other staff understand was difficult" }\end{array}$ & $\begin{array}{l}\text { Autonomy: "The titration in dextrose } \\
\text { containing fluids is less defined allowing } \\
\text { more clinical judgment to be exercised." }\end{array}$ \\
\hline Everything & $\begin{array}{l}\text { Effective: "It had a good initial treatment plan } \\
\text { with the 2 bag fluid system and initial labs." } \\
\text { "it worked well, and you saw results" }\end{array}$ \\
\hline $\begin{array}{l}\text { Precise: Straight-forward; black and white; } \\
\text { exact; exact guidelines; info on starting and } \\
\text { stopping }\end{array}$ & $\begin{array}{l}\text { Easy to use: "Ease of use and following" "It } \\
\text { was straightforward and able to be easily } \\
\text { followed by patient lab values. Drip changes } \\
\text { were able to be made based on these lab } \\
\text { values in an easy to understand way." "Ease } \\
\text { to follow" "Less qualifications for pt being } \\
\text { corrected" "Ease of use" }\end{array}$ \\
\hline $\begin{array}{l}\text { Easy to use: Easy to follow; allows for } \\
\text { autonomy }\end{array}$ & $\begin{array}{l}\text { Adaptable: "You were able to go back on NS } \\
\text { if your sugar was climbing" }\end{array}$ \\
\hline Not sure & $\begin{array}{l}\text { Familiarity: "Familiarity. Takes some time } \\
\text { and practice to become comfortable." }\end{array}$ \\
\hline
\end{tabular}

Question: What is something you would change about the previous protocol?

\begin{tabular}{|l|l|}
\hline Initial survey & Final survey \\
\hline $\begin{array}{l}\text { No changes: "No" "“Not much I liked it" } \\
\text { "Nothing" }\end{array}$ & Needs updated: "Urine analysis" \\
\hline $\begin{array}{l}\text { Too difficult/bad format: "It needed to be } \\
\text { easier for the new RNs to read and } \\
\text { understand" "Add in the specific fluid rate } \\
\text { breakdowns; as you did on the new version - } \\
\text { love it" "The way it is written. Flow-chart or } \\
\text { some type of simplified structure would be } \\
\text { more helpful" "All of the things that you have } \\
\text { changed to develop the new protocol. Easy to } \\
\text { read, user friendly, up-to-date evidenced } \\
\text { based practice" "More clear instructions for } \\
\text { residents" }\end{array}$ & $\begin{array}{l}\text { Too difficult/bad format/ too wordy: } \\
\text { treatment of cerebral edema." "It is difficult } \\
\text { to follow and some of the flow chart does not } \\
\text { about changing rates based on glucose } \\
\text { results" "No set parameters for changing } \\
\text { fluids" "The previous protocol was very } \\
\text { wordy and difficult to follow" "Make it easier } \\
\text { to follow" "It is difficult to follow and some } \\
\text { of the flow chart does not flow well" "The } \\
\text { previous protocol was very wordy and } \\
\text { difficult to follow" "Just too wordy. But } \\
\text { otherwise I liked it" "Diagram is illogical in } \\
\text { certain situations" "User friendly }\end{array}$ \\
\begin{tabular}{l} 
No changes: "Nothing" \\
\hline
\end{tabular} \\
\hline Needs updated to current EBP
\end{tabular}


Not used universally by staff: "that it would be used universally by all staff"
Not used universally by staff: "Better adherence by attendings" "The consistency in which the providers use it."

Question: What is something you like about the new protocol?

\begin{tabular}{|l|l|}
\hline Initial survey & Final survey \\
\hline $\begin{array}{l}\text { Good format: "The easy to read weight } \\
\text { chart." "Format" "The fluid rate chart" "Easy } \\
\text { to follow" "User friendly, evidence based" } \\
\text { "Easy to follow" "Verbiage" "Fluid chart" }\end{array}$ & $\begin{array}{l}\text { Updated/EBP: "Urine analysis D/C on } \\
\text { ongoing urine. There is a flowsheet for visual } \\
\text { learners." "Better focus on warning signs and } \\
\text { treatment for cerebral edema" }\end{array}$ \\
\hline $\begin{array}{l}\text { Evidence-based: "User friendly, evidence } \\
\text { based" }\end{array}$ & $\begin{array}{l}\text { Good format: "Urine analysis D/C on } \\
\text { ongoing urine. There is a flowsheet for visual } \\
\text { learners." "There are more clear guidelines } \\
\text { for nursing" "More specific" "MIVF calcs" "I } \\
\text { like that the new protocol is less wordy and } \\
\text { more straight forward. It's easy to follow. I } \\
\text { like the charts that are with it." "Flowchart" } \\
\text { "Less wordy. Easy to read and follow" } \\
\text { "Specific outlined instructions" }\end{array}$ \\
\hline Unsure: "NA" "Haven't used" & Nothing \\
\hline
\end{tabular}

Question: What is something you would change about the new protocol?

\begin{tabular}{|c|c|}
\hline Initial survey & Final survey \\
\hline \multirow[t]{2}{*}{ Nothing } & $\begin{array}{l}\text { Does not correct for increasing blood } \\
\text { glucose: "It does not have what to do if your } \\
\text { glucose increases again after you have been } \\
\text { steadily decreasing all shift." "There are } \\
\text { times where the two pathways do not address } \\
\text { the issue at hand. I have also had a patient } \\
\text { where the blood glucose was falling at a } \\
\text { perfect rate each hour and yet when my } \\
\text { glucose is right below } 300 \text { I go up on my } \\
\text { dextrose and the patient stays with glucoses } \\
>250 \text { and we end up going back to what we } \\
\text { did before with titrating and not increasing } \\
\text { dextrose until the glucose gets below } 100 . \text { "It } \\
\text { only ever tells you to increase dextrose. Never } \\
\text { able to go down on dextrose" }\end{array}$ \\
\hline & $\begin{array}{l}\text { Too much titration: "The goal for serum } \\
\text { glucose is quite narrow. From past } \\
\text { experience, there is fluctuation both up and } \\
\text { down when infusing at the some dextrose }\end{array}$ \\
\hline
\end{tabular}




\begin{tabular}{|c|c|}
\hline & $\begin{array}{l}\text { concentration. The new protocol requires } \\
\text { more titration which has caused glucose } \\
\text { levels to swing faster. Also, by having to } \\
\text { titrate frequently, there is an increase in } \\
\text { opportunity for med errors." }\end{array}$ \\
\hline & $\begin{array}{l}\text { Not followed universally by staff: "Just } \\
\text { make sure nursing is following the protocol. } \\
\text { I've had a couple calls about nursing asking } \\
\text { me what changes to make. Always happy to } \\
\text { help and take calls but we need to be sure to } \\
\text { continue following protocol" "The } \\
\text { consistency in which providers use it." }\end{array}$ \\
\hline & $\begin{array}{l}\text { Not specific enough: "DKA changes aren't } \\
\text { always black \& white, so I'd have exceptions } \\
\text { listed" "Become more aware of new labs" } \\
\text { "Guidelines for being corrected" }\end{array}$ \\
\hline & $\begin{array}{l}\text { Not familiar enough to make suggestions: } \\
\text { "I have only used it twice so nothing as of } \\
\text { now" }\end{array}$ \\
\hline & $\begin{array}{l}\text { Suggestion for tx change for cerebral } \\
\text { edema: "I would list } 3 \% \mathrm{NaCl} \text { as the first line } \\
\text { treatment for cerebral edema and Mannitol as } \\
\text { a second line choice due to its side effects." }\end{array}$ \\
\hline
\end{tabular}

Question: From what you've seen, do you prefer the old DKA protocol or the new DKA protocol? Why?

\begin{tabular}{|l|l|}
\hline Initial survey & Final survey \\
\hline Unsure/no preference: "Same" "Unsure. & $\begin{array}{l}\text { New: "New protocol gives more clear } \\
\text { instructions for the team." "New, easier } \\
\text { guidelines and I like how the math is clearly } \\
\text { new one yet" "Not sure yet. Change is } \\
\text { difficult. Just need to use it more" "I have no } \\
\text { preference, but am excited to learn something } \\
\text { new" "Both seem easy to use" "Both works" } \\
\text { "Not sure" "Haven't used the new protocol } \\
\text { follow" "New, ease in calcs" "New" "New. } \\
\text { Flowchart is easy to follow" "New protocol: } \\
\text { helps nursing with fluid rate calculations" } \\
\text { "New, as it is cleaned up and easier to follow" } \\
\text { of use." "New" "New just for the readability } \\
\text { and ease of use" "new is more detailed, so the } \\
\text { new one" "New is more user friendly" "New, } \\
\text { recent research and best practice" }\end{array}$ \\
$\begin{array}{l}\text { Old: "Old. There is more room for clinical } \\
\text { judgement and I have better glucose control } \\
\text { with less titration." "Old DKA because I think } \\
\text { you could obtain true 1.5 MIVF instead of } \\
\text { rounding to the nearest weight." "Old, I feel } \\
\text { that the new leads practice to make changes } \\
\text { that are not necessarily correct." "Old DKA } \\
\text { protocol: easy to follow, works well" "I prefer } \\
\text { the old protocol because I feel like it is more } \\
\text { precise, but I do like the flow chart of the new }\end{array}$ \\
\hline
\end{tabular}




\begin{tabular}{|l|l|}
\hline & $\begin{array}{l}\text { one." "Old" "old" "Old for reasons previously } \\
\text { stated" "Old: it seems they correct quicker } \\
\text { based off the old protocol" "OLD" "At this } \\
\text { point, the old. With residents rotating every } \\
\text { month, it is hard to get consistency with use } \\
\text { of protocol. With the old protocol being well } \\
\text { established, at least the nurses can have more } \\
\text { confidence in using it, therefore teaching new } \\
\text { residents and new nurses to use." }\end{array}$ \\
\hline Old: "Old - just what I'm used to" & \\
\hline
\end{tabular}




\section{References}

Abbas, Q., Arbab, S., Anwar ul Haque, A., \& Humayun, K.N. (2018). Spectrum of complications of severe DKA in children in pediatric intensive care unit. Pakistan Journal of Medical Sciences, 34(1), 106-109. doi: 10.12669/pjms.341.13875

Al Nemri, A.A., Amer, Y.S., Gasim, H., Osman, M.E., Aleyadhy, A., Al Otaibi, H.,...\& Mohamed, S. (2017). Substantial reduction in hospital stay of children and adolescents with diabetic ketoacidosis after implementation of Clinical Practice Guidelines in a university hospital in Saudi Arabia. Journal of Evaluation in Clinical Practice, 23(1), 173177. Retrieved June 30, 2019 from https://onlinelibrary-wileycom.www.libproxy.wvu.edu/doi/full/10.1111/jep.12661

Bağ, Ö., Tunç, S., Nalbantoğlu, Ö., Ecevit, Ç., Öztürk, A., Özkan, B., \& Demir, K. (2017). Higher-than conventional subcutaneous regular insulin doses in diabetic ketoacidosis in children and adolescents. Journal of Clinical Research in Pediatric Endocrinology, 9(2), 132-137. Retrieved June 29, 2019 from https://doiorg.www.libproxy.wvu.edu/10.4274/jcrpe.3925

Bakes, K., Haukoos, J. S., Deakyne, S. J., Hopkins, E., Easter, J., McFann, K., ... Rewers, A. (2016). Effect of volume of fluid resuscitation on metabolic normalization in children presenting in diabetic ketoacidosis: A randomized controlled trial. Journal of Emergency Medicine (0736-4679), 50(4), 551-559. https://doiorg.www.libproxy.wvu.edu/10.1016/j.jemermed.2015.12.003

Barski, L., Brandstaetter, E., Sagy, I., \&Jotkowitz, A. (2018). Basal insulin for the management of diabetic ketoacidosis. European Journal of Internal Medicine, 47, 14-16. Retrieved June 29, 2019 from https://doi-org.www.libproxy.wvu.edu/10.1016/j.ejim.2017.08.025 
Benoit, S.R., Zhang, Y., Geiss, L.S., Gregg, E.W., \& Albright, A. (2018). Trends in diabetic ketoacidosis hospitalizations and in-hospital mortality - United States 2000-2014. MMWR Morb Mortal Wkly Rep 2018, 67, 362-365.

DOI: $\underline{\text { http://dx.doi.org/10.15585/mmwr.mm6712a3 }}$

Bowen, D.J., Kreuter, M., Spring, B., Coft-Woerpel, L., Linnan, L., Weiner, D., Bakken, S., Kaplan, C.P., Squiers, L., Fabrizio, C., \& Fernandez, M. (2009). How we design feasible studies. American Journal of Preventive Medicine, 36(5), 452-457. Doi:

10.1016/j.amepre.2009.02.002.

Cohen, M., Leibovitz, N., Shilo, S., Zuckerman, L. N., Shavit, I., \& Shehadeh, N. (2017).

Subcutaneous regular insulin for the treatment of diabetic ketoacidosis in children.

Pediatric Diabetes, 18(4), 290-296. Retrieved June 29, 2019 from https://doi-

org.www.libproxy.wvu.edu/10.1111/pedi.12380

Corporate Finance Institute (2015). SMART goal: Specific, measurable, attainable, realistic, timely. https://corporatefinanceinstitute.com/resources/knowledge/other/smartgoal/

Desai, D., Mehta, D., Mathias, P., Menon, G., \&Schubart, U.K. (2018). Health care utilization and burden of diabetic ketoacidosis in the U.S. over the past decade: A nationwide analysis. Diabetes Care 2018 August, 41(8), 1631-1638. Retrieved July 12, 2019 from https://care.diabetesjournals.org/content/41/8/1631.long

Dhochak, N., Jayashree, M., \&Singhi, S. (2018). A randomized controlled trial of one bag vs. two bag system of fluid delivery in children with diabetic ketoacidosis: Experience from a developing country. Journal of Critical Care, 43, 340-345. Retrieved June 30, 2019 from https://doi.org/10.1016/j.jcrc.2017.09.175 
Duffy, J. R., \& Hoskins, L. M. (2003). The Quality-Caring Model: blending dual paradigms. ANS. Advances in Nursing Science, 26(1), 77-88. Retrieved February 2, 2020 from

http://search.ebscohost.com.www.libproxy.wvu.edu/login.aspx?direct=true $\& \mathrm{db}=\mathrm{cmedm}$ $\underline{\mathrm{AN}}=12611432$

Flood, K., Nour, M., Holt, T., Cattell, V., Krochak, C., \& Inman, M. (2019). Implementation and evaluation of a diabetic ketoacidosis order set in pediatric type 1 diabetes at a tertiary care hospital: A Quality-Improvement Initiative. Canadian Journal of Diabetes, 43(5), 297-303. Retrieved July 13, 2019 from https://www-clinicalkeycom.www.libproxy.wvu.edu/\#!/content/playContent/1-s2.0$\underline{\mathrm{S} 1499267118303794 ? \text { scrollTo }=\% 23 \mathrm{~h} 10000383}$

Harrison, V. S., Rustico, S., Palladino, A. A., Ferrara, C., \& Hawkes, C. P. (2017). Glargine co administration with intravenous insulin in pediatric diabetic ketoacidosis is safe and facilitates transition to a subcutaneous regimen. Pediatric Diabetes, 18(8), 742-748. Retrieved June 29, 2019 from https://doi-org.www.libproxy.wvu.edu/10.1111/pedi.12462

Horvat, C. M., Ismail, H. M., Au, A. K., Garibaldi, L., Siripong, N., Kantawala, S., ... Clark, R. S. B. (2018). Presenting predictors and temporal trends of treatment-related outcomes in diabetic ketoacidosis. Pediatric Diabetes, 19(5), 985-992. Retrieved June 29, 2019 from https://doi-org.www.libproxy.wvu.edu/10.1111/pedi.12663

Kim, S.Y. (2015). Endocrine and metabolic emergencies in children: hypocalcemia, hypoglycemia, adrenal insufficiency, and metabolic acidosis including diabetic ketoacidosis. Annals of Pediatric Endocrinology \& Metabolism, 20(4), 179-186. Retrieved July 8, 2019 from https://www-ncbi-nlm-nih- 
gov.www.libproxy.wvu.edu/pmc/articles/PMC4722156/

Koves, I.H., Leu, M.G., Spencer, S., Popalisky, J.C., Drummond, K., Beardsley, E., ... \& Zimmerman, J.J. (2014). Improving care for pediatric diabetic ketoacidosis. Pediatrics, 134(3), 848-856. DOI: $10.1542 /$ peds.2013-3764

Kuppermann, N., Ghetti, S., Schunk, J. E., Stoner, M. J., Rewers, A., McManemy, J. K., ... Dean, J. M. (2018). Clinical trial of fluid infusion rates for pediatric diabetic ketoacidosis. New England Journal of Medicine, 378(24), 2275-2287. Retrieved June 29, 2019 from https://doi-org.www.libproxy.wvu.edu/10.1056/NEJMoa1716816

Long, B., \&Koyfman, A. (2017). Emergency medicine myths: Cerebral edema in pediatric diabetic ketoacidosis and intravenous fluids. Journal of Emergency Medicine (07364679), 53(2), 212-221. Retrieved June 29, 2019 from https://doiorg.www.libproxy.wvu.edu/10.1016/j.jemermed.2017.03.014

Mayer-Davis, E., Lawrence, J.M., Dabelea, D., Divers, J., Isom, S., Dolan, L., ... Wagenknecht, L. (2017). Incidence trends of type 1 and type 2 diabetes among youths, 2002-2012. New England Journal of Medicine, 376(15), 1419-1429. doi: 10.1056/NEJMoa1610187

Nallasamy, K., Jayashree, M., Singhi, S., \& Bansal, A. (2014). Low-dose vs standard-dose insulin in pediatric diabetic ketoacidosis: a randomized clinical trial. JAMA Pediatrics, 168(11), 999-1005. Retrieved June 30, 2019 from https://jamanetworkcom.www.libproxy.wvu.edu/journals/jamapediatrics/fullarticle/1909800

Patel, A., Singh, D., Bhatt, P., Thakkar, B., Akingbola, O. A., \& Srivastav, S. K. (2015). Incidence, trends, and outcomes of cerebral edema among children with diabetic ketoacidosis in the United States. Clinical Pediatrics, 55(10), 943-951. Retrieved June 30, 2019 from https://doi-org.www.libproxy.wvu.edu/10.1177/0009922815617975 
Peeters, E., van IJperen, W.J., Robertson, L., Royle, P., \& van IJperen, E. Sr. (2015). Analysis of the safety and efficacy of diabetic ketoacidosis management in a community general hospital, 2001-2010: A descriptive study. Scottish Medical Journal, 60(3), 121-125. Retrieved May 19, 2019 from https://illiad.lib.wvu.edu/illiad.dll? Action $=10 \&$ Form $=75 \&$ Value $=858028$

Razavi, Z., Maher, S., \&Fredmal, J. (2018). Comparison of subcutaneous insulin aspart and intravenous regular insulin for the treatment of mild and moderate diabetic ketoacidosis in pediatric patients. Endocrine, 61(2), 267-274. Retrieved June 30, 2019 from DOI:

\section{$\underline{10.1007 / \mathrm{s} 12020-018-1635-\mathrm{Z}}$}

Santi, M., Lava, S.A.G., Camozzi, P., Giannini, O., Milani, G.P., Simonetti, G.D., ... \& Fare, P.B. (2015). The great fluid debate: saline or so-called "balanced" salt solutions? Italian Journal of Pediatrics, 41(47), 1-5. Retrieved July 8, 2019 from https://ijponline.biomedcentral.com/articles/10.1186/s13052-015-0154-2

Shafi, O. \& Kumar, V. (2018). Initial fluid therapy in pediatric diabetic ketoacidosis: A comparison of hypertonic saline solution and normal saline solution. Pediatric Endocrinology Diabetes and Metabolism, 24(2), 56-64. Retrieved July 8, 2019 from https://pdfs.semanticscholar.org/b3a5/37eeflf6eedfb1753b7bb8b0ed4db8f4f7e3.pdf

Shankar, V., Haque, A., Churchwell, K. B., \& Russell, W. (2007). Insulin glargine supplementation during early management phase of diabetic ketoacidosis in children. Intensive Care Medicine, 33(7), 1173-1178. https://doiorg.www.libproxy.wvu.edu/10.1007/s00134-007-0674-3

Skitch, S. A., \&Valani, R. (2015). Treatment of Pediatric Diabetic Ketoacidosis in Canada: A review of treatment protocols from Canadian pediatric emergency departments. CJEM: 
Canadian Journal of Emergency Medicine, 17(6), 656-661. Retrieved June 29, 2019 from https://doi-org.www.libproxy.wvu.edu/10.1017/cem.2015.56

Stevens, K. R. (2012). Star model of ebp: Knowledge transformation. Academic Center for Evidence-based Practice. The University of Texas Health Science Center at San Antonio. Retrieved June 3, 2019 from https://nursing.uthscsa.edu/onrs/starmodel/star-model.asp

von Oettingen, J. E., Rhodes, E. T., \&Wolfsdorf, J. I. (2018). Resolution of ketoacidosis in children with new onset diabetes: Evaluation of various definitions. Diabetes Research \& Clinical Practice, 135, 76-84.Retrieved June 29, 2019 from https://doiorg.www.libproxy.wvu.edu/10.1016/j.diabres.2017.09.011

Wolfsdorf, J. I., Glaser, N., Agus, M., Fritsch, M., Hanas, R., Rewers, A., ... Codner, E. (2018). ISPAD Clinical Practice Consensus Guidelines 2018: Diabetic ketoacidosis and the hyperglycemic hyperosmolar state. Pediatric Diabetes, 19, 155-177. Retrieved June 29, 2019 from https://doi-org.www.libproxy.wvu.edu/10.1111/pedi.12701

Yung, M., Letton, G., \& Keeley, S. (2017). Controlled trial of Hartmann’s solution versus 0.9\% saline for diabetic ketoacidosis. Journal of Paediatrics and Child Health, 53(1), 12-17. Retrieved July 8, 2019 from https://onlinelibrary-wileycom.www.libproxy.wvu.edu/doi/full/10.1111/jpc.13436 